\title{
LR-Preinvex Interval-Valued Functions and Riemann-Liouville Fractional Integral Inequalities
}

\author{
Muhammad Bilal Khan ${ }^{1, *(\mathbb{C})}$, Muhammad Aslam Noor ${ }^{1}\left(\mathbb{D}\right.$, Thabet Abdeljawad ${ }^{2,3, * \mathbb{C}}$, Abd Allah A. Mousa ${ }^{4}(\mathbb{D}$, \\ Bahaaeldin Abdalla ${ }^{2}$ (D) and Safar M. Alghamdi 4 (D) \\ 1 Department of Mathematics, COMSATS University Islamabad, Islamabad 44000, Pakistan; \\ aslamnoor@comsats.edu.pk \\ 2 Department of Mathematics and Sciences, Prince Sultan University, \\ P.O. Box 66833, Riyadh 11586, Saudi Arabia; babdallah@psu.edu.sa \\ 3 Department of Medical Research, China Medical University, Taichung 40402, Taiwan \\ 4 Department of Mathematics and Statistics, College of Science, Taif University, \\ P.O. Box 11099, Taif 21944, Saudi Arabia; a.mousa@tu.edu.sa (A.A.A.M.); S.m.alghamdi@tu.edu.sa (S.M.A.) \\ * Correspondence: bilal42742@gmail.com (M.B.K.); tabdeljawad@psu.edu.sa (T.A.)
}

Citation: Khan, M.B.; Noor, M.A.; Abdeljawad, T.; Mousa, A.A.A.; Abdalla, B.; Alghamdi, S.M. LR-Preinvex Interval-Valued Functions and Riemann-Liouville Fractional Integral Inequalities. Fractal Fract. 2021, 5, 243. https:// doi.org/10.3390/fractalfract5040243

Academic Editor: Mahamadi Warma

Received: 20 October 2021

Accepted: 20 November 2021

Published: 29 November 2021

Publisher's Note: MDPI stays neutral with regard to jurisdictional claims in published maps and institutional affiliations.

Copyright: (c) 2021 by the authors. Licensee MDPI, Basel, Switzerland. This article is an open access article distributed under the terms and conditions of the Creative Commons Attribution (CC BY) license (https:// creativecommons.org/licenses/by/ $4.0 /)$.

\begin{abstract}
Convexity is crucial in obtaining many forms of inequalities. As a result, there is a significant link between convexity and integral inequality. Due to the significance of these concepts, the purpose of this study is to introduce a new class of generalized convex interval-valued functions called LR-preinvex interval-valued functions (LR-preinvex $I-V-F s$ ) and to establish Hermite-Hadamard type inequalities for LR-preinvex $I-V-F s$ using partial order relation $\left(\leq_{p}\right)$. Furthermore, we demonstrate that our results include a large class of new and known inequalities for LR-preinvex interval-valued functions and their variant forms as special instances. Further, we give useful examples that demonstrate usefulness of the theory produced in this study. These findings and diverse approaches may pave the way for future research in fuzzy optimization, modeling, and interval-valued functions.
\end{abstract}

Keywords: LR-preinvex interval-valued function; fractional integral operator; Hermite-Hadamard type inequality; Hermite-Hadamard Fejér type inequality

\section{Introduction}

Fractional calculus traces back to the seventeenth century, when G.W. Leibniz and the Marquis de l'Hospital first discussed semi-derivatives. This question prompted several eminent mathematicians to study modern ideas on the subject. The theory of fractional calculus evolved greatly in the late nineteenth century, and currently encompasses mathematics, physics, viscoelasticity, rheology, chemistry, statistical physics, and electrical and mechanical engineering.

The number of papers on the use of integral inequalities in mathematical analysis has increased exponentially. Integral operators such as Riemann-Liouville, Caputo, Katugampola, and Caputo-Fabrizio have been constructed in recent years utilizing a variety of fractional-order operator definitions. Several variants of well-known inequalities of Hermite-Hadamard, Hardy, Opial, Ostrowski, and Grüss have been found using these integrals (see [1-10]).

On the other hand, Costa [11] recently discovered Jensen's type inequality for intervalvalued functions $(I-V-F s)$ and fuzzy-interval-valued functions ( $F-I-V-F s)$. Costa and RomanFlores $[12,13]$ discussed the attributes of several forms of inequalities for $F-I-V-F$ and $I-V-F$. Gronwall type inequality was developed for $I-V-F s$ by Roman-Flores et al. [14]. Furthermore, Chalco-Cano et al. [15,16] used the generalized Hukuhara derivative to present Ostrowski-type inequalities for $I-V-F s$ and gave applications in numerical integration of $I-V-F$. New versions of Jensen's inequality for strongly convex and convex functions were provided by Nikodem et al. [17] and Matkowski and Nikodem [18]. Zhao et al. [19,20] used 
$I-V$-Fs to derive Chebyshev, Jensen's, and $H \cdot H$ type inequalities. Zhang et al. [21] recently used a pseudo-order relation to expand Jensen's inequalities and establish a novel version of Jensen's inequalities for set-valued and fuzzy-set-valued functions. Budak [22] then used an inclusion relation to establish interval-valued fractional Riemann-Liouville $H \cdot H$ inequality for convex $I-V-F$. See [23-26] and the references therein for more information.

Khan et al. [27] recently used fuzzy order relation to establish a new class of convex $F-I-V$-Fs known as $\left(h_{1}, h_{2}\right)$-convex F-I-V-Fs, as well as a novel version of the $H \cdot H$ type inequality for $\left(h_{1}, h_{2}\right)$-convex $F-I-V$ - $F$ s that incorporates the FI Riemann integral. Khan et al. went a step further by providing new convex and extended convex $F-I-V-F$ classes, as well as new fractional $H \cdot H$ type and $H \cdot H$ type inequalities for $\left(h_{1}, h_{2}\right)$-preinvex $F-I-V-F$ [28] log-s-convex F-I-V-Fs in the second sense [29], and harmonically convex F-I-V-Fs [30] and the references therein. We refer to the readers for further analysis of literature on the applications and properties of FI, and inequalities and generalized convex fuzzy mappings, see [31-54] and the references therein.

Motivated and inspired by ongoing research work, we have introduced the new generalization of convex functions is known as LR-preinvex $I-V$-Fs using partial order relation. With the support of this class, we have derived the new versions of HermiteHadamard inequalities using Riemann-Liouville fractional operators. Moreover, we have discussed the exceptional cases as applications of this study.

\section{Preliminaries}

Let $\mathbb{R}_{I}$ be the collection of all closed and bounded intervals of $\mathbb{R}$ that is $\mathbb{R}_{I}=\left\{\left[Z_{*}, Z^{*}\right]: Z_{*}, Z^{*} \in \mathbb{R}\right.$ and $\left.Z_{*} \leq Z^{*}\right\}$. If $Z_{*} \geq 0$, then $\left[Z_{*}, Z^{*}\right]$ is called positive interval. The set of all positive interval is denoted by $\mathbb{R}_{I}^{+}$and defined as $\mathbb{R}_{I}^{+}=\left\{\left[Z_{*}, Z^{*}\right] \mid\left[Z_{*}, Z^{*}\right] \in \mathbb{R}_{I}\right.$ and $\left.Z_{*} \geq 0\right\}$.

We now discuss some properties of intervals under the arithmetic operations addition, multiplication, and scalar multiplication. if $\left[W_{*}, W^{*}\right],\left[Z_{*}, Z^{*}\right] \in \mathbb{R}_{I}$ and $\rho \in \mathbb{R}$, then arithmetic operations are defined by

$$
\left[W_{*}, W^{*}\right]+\left[Z_{*}, Z^{*}\right]=\left[W_{*}+Z_{*}, W^{*}+Z^{*}\right],
$$

$\left[W_{*}, W^{*}\right] \times\left[Z_{*}, Z^{*}\right]=\left[\min \left\{W_{*} Z_{*}, W^{*} Z_{*}, W_{*} Z^{*}, W^{*} Z^{*}\right\}, \max \left\{W_{*} Z_{*}, W^{*} Z_{*}, W_{*} Z^{*}, W^{*} Z^{*}\right\}\right]$,

$$
\rho .\left[W_{*}, W^{*}\right]=\left\{\begin{array}{l}
{\left[\rho W_{*}, \rho W^{*}\right] \text { if } \rho \geq 0,} \\
{\left[\rho W^{*}, \rho W_{*}\right] \text { if } \rho<0 .}
\end{array}\right.
$$

For $\left[W_{*}, W^{*}\right],\left[Z_{*}, Z^{*}\right] \in \mathbb{R}_{I}$, the inclusion " $\subseteq$ " is defined by

$$
\left[W_{*}, W^{*}\right] \subseteq\left[Z_{*}, Z^{*}\right] \text {, if and only if, } Z_{*} \leq W_{*}, W^{*} \leq Z^{*} .
$$

Remark 1. [21] (i) The relation " $\leq_{p}$ " defined on $\mathbb{R}_{I}$ by

$$
\left[W_{*}, W^{*}\right] \leq_{p}\left[Z_{*}, Z^{*}\right] \text { if and only if, } W_{*} \leq Z_{*}, W^{*} \leq Z^{*},
$$

for all $\left[W_{*}, W^{*}\right],\left[Z_{*}, Z^{*}\right] \in \mathbb{R}_{I}$ is a pseudo-order relation. The relation $\left[W_{*}, W^{*}\right] \leq_{p}\left[Z_{*}, Z^{*}\right]$ coincident to $\left[W_{*}, W^{*}\right] \leq\left[Z_{*}, Z^{*}\right]$ on $\mathbb{R}_{I}$, where " $\leq$ " is paritail order relation on $\mathbb{R}_{I}$.

(ii) It can be easily seen that " $\leq_{p}$ " looks like "left and right" on the real line $\mathbb{R}$, so we call " $\leq_{p}$ " is "left and right" (or "LR" order, in short).

The concept of Riemann integral for $I-V-F$ first introduced by Moore [42] is defined as follow:

Theorem 1. [42] If $\mathfrak{Q}:[t, s] \subset \mathbb{R} \rightarrow \mathbb{R}_{I}$ is an I-V-F on such that $\mathfrak{Q}(\omega)=\left[\mathfrak{Q}_{*}(\omega), \mathfrak{Q}^{*}(\omega)\right]$. Then $\mathfrak{Q}$ is interval Riemann integrable over $[t, s]$ if and only if, $\mathfrak{Q}_{*}$ and $\mathfrak{Q}^{*}$ both are Riemann integrable over $[t, s]$ such that

$$
(I R) \int_{t}^{s} \mathfrak{Q}(\omega) d \omega=\left[(R) \int_{t}^{s} \mathfrak{Q}_{*}(\omega) d \omega,(R) \int_{t}^{s} \mathfrak{Q}^{*}(\omega) d \omega\right],
$$


where the notions $(I R) \int$ and $(R) \int$ represent the interval Riemann and classical Riemann integrals operators when integrant are interval-valued functions and single valued functions, respectively. The collection of all Riemann integrable real valued functions and Riemann integrable $I-V-F$ is denoted by $\mathcal{R}_{[t, s]}$ andi $\mathcal{R}_{[t, s]}$, respectively.

Buduk et al. and Lupulescu [22,41] introduced the following interval RiemannLiouville fractional integral operators:

Let $\alpha>0$ and $L\left([t, s], \mathbb{R}_{I}^{+}\right)$be the collection of all Lebesgue measurable $I-V$-Fs on $[t, s]$. Then the interval left and right Riemann-Liouville fractional integral of $\mathfrak{Q} \in L\left([t, s], \mathbb{R}_{I}^{+}\right)$ with order $\alpha>0$ are defined by

$$
\mathcal{I}_{t^{+}}^{\alpha} \mathfrak{Q}(\omega)=\frac{1}{\Gamma(\alpha)} \int_{t}^{\omega}(\omega-\sigma)^{\alpha-1} \mathfrak{Q}(\sigma) d \sigma, \quad(\omega>t),
$$

and

$$
\mathcal{I}_{s^{-}}^{\alpha} \mathfrak{Q}(\omega)=\frac{1}{\Gamma(\alpha)} \int_{\omega}^{s}(\sigma-\omega)^{\alpha-1} \mathfrak{Q}(\sigma) d \sigma, \quad(\omega<s)
$$

respectively, where $\Gamma(\omega)=\int_{0}^{\infty} \sigma^{\omega-1} e^{-\sigma} d \sigma$ is the Euler varGamma function.

Definition 1. [21] The I-V-F $\mathfrak{Q}: K \rightarrow \mathbb{R}_{I}^{+}$is said to be $L R$-convex-I-V-F on convex set $K$ if for all $\omega, \mathcal{Z} \in K$ and $\sigma \in[0,1]$ we have

$$
\mathfrak{Q}(\sigma \omega+(1-\sigma) \mathcal{Z}) \leq_{p} \sigma \mathfrak{Q}(\omega)+(1-\sigma) \mathfrak{Q}(\mathcal{Z}),
$$

If inequality (5) is reversed, then $\mathfrak{Q}$ is said to be LR-concave on $K . \mathfrak{Q}$ is affine if and only if, it is both LR-convex and LR-concave.

Motivated and inspired by existing research, we introduce a novel extension of convex functions called as LR-preinvex $I-V-F s$ utilizing partial order relation. Then, with the assistance of this class, we develop new Hermite-Hadamard inequalities utilizing RiemannLiouville fractional operators. Moreover, we highlight the exceptional cases that arise as a result of our research.

Definition 2. The I-V-F $\mathfrak{Q}: K \rightarrow \mathbb{R}_{I}^{+}$is said to be $L R$-preinvex-I-V-F on invex set $K$ if for all $\omega, \mathcal{Z} \in K$ and $\sigma \in[0,1]$ we have

$$
\mathfrak{Q}(\omega+(1-\sigma) \varphi(\mathcal{Z}, \omega)) \leq_{p} \sigma \mathfrak{Q}(\omega)+(1-\sigma) \mathfrak{Q}(\mathcal{Z}),
$$

where $\varphi: K \times K \rightarrow \mathbb{R}$. If inequality (4) is reversed, then $\mathfrak{Q}$ is said to be LR-preincave on $K . \mathfrak{Q}$ is affine if and only if it is both LR-preinvex and LR-preincave.

Theorem 2. Let $K$ be an invex set and $\mathfrak{Q}: K \rightarrow \mathbb{R}_{I}^{+}$be a $I-V-F$ such that

$$
\mathfrak{Q}(\omega)=\left[\mathfrak{Q}_{*}(\omega), \mathfrak{Q}^{*}(\omega)\right], \forall \omega \in K .
$$

for all $\omega \in K$. Then $\mathfrak{Q}$ is $L R$-preinvex-I-V-F on $K$, if and only if, $\mathfrak{Q}_{*}(\omega)$ and $\mathfrak{Q}^{*}(\omega)$ both are preinvex functions.

Proof. Assume that $\mathfrak{Q}_{*}, \mathfrak{Q}^{*}$ are preinvex functions. Then, for all $\omega, \mathcal{Z} \in K, \sigma \in[0,1]$, we have

$$
\mathfrak{Q}_{*}(\omega+(1-\sigma) \varphi(\mathcal{Z}, \omega)) \leq \sigma \mathfrak{Q}_{*}(\omega)+(1-\sigma) \mathfrak{Q}_{*}(\mathcal{Z}),
$$

and

$$
\mathfrak{Q}^{*}(\omega+(1-\sigma) \varphi(\mathcal{Z}, \omega)) \leq \sigma \mathfrak{Q}^{*}(\omega)+(1-\sigma) \mathfrak{Q}^{*}(\mathcal{Z}) .
$$


From Definition 2 and order relation $\leq p$, we have

$$
\begin{array}{r}
{\left[\mathfrak{Q}_{*}(\omega+(1-\sigma) \varphi(\mathcal{Z}, \omega)), \mathfrak{Q}^{*}(\omega+(1-\sigma) \varphi(\mathcal{Z}, \omega))\right]} \\
\leq_{p}\left[\sigma \mathfrak{Q}_{*}(\omega), \sigma \mathfrak{Q}^{*}(\omega)\right]+\left[(1-\sigma) \mathfrak{Q}_{*}(\mathcal{Z}),(1-\sigma) \mathfrak{Q}^{*}(\mathcal{Z})\right],
\end{array}
$$

that is

$$
\mathfrak{Q}(\omega+(1-\sigma) \varphi(\mathcal{Z}, \omega)) \leq_{p} \sigma \mathfrak{Q}(\omega)+(1-\sigma) \mathfrak{Q}(\mathcal{Z}), \forall \omega, \mathcal{Z} \in K, \sigma \in[0,1] .
$$

Hence, $\mathfrak{Q}$ is LR-preinvex-I-V-F. we have

Conversely, let $\mathfrak{Q}$ be a LR-preinvex-I-V-F. Then, for all $\omega, \mathcal{Z} \in K$ and $\sigma \in[0,1]$,

$$
\mathfrak{Q}(\omega+(1-\sigma) \varphi(\mathcal{Z}, \omega)) \leq_{p} \sigma \mathfrak{Q}(\omega)+(1-\sigma) \mathfrak{Q}(\mathcal{Z}),
$$

that is

$\left[\mathfrak{Q}_{*}(\omega+(1-\sigma) \varphi(\mathcal{Z}, \omega)), \mathfrak{Q}^{*}(\omega+(1-\sigma) \varphi(\mathcal{Z}, \omega))\right] \leq_{p}\left[\sigma \mathfrak{Q}_{*}(\omega), \sigma \mathfrak{Q}^{*}(\omega)\right]+\left[(1-\sigma) \mathfrak{Q}_{*}(\mathcal{Z}),(1-\sigma) \mathfrak{Q}^{*}(\mathcal{Z})\right]$.

It follows that

$$
\mathfrak{Q}_{*}(\omega+(1-\sigma) \varphi(\mathcal{Z}, \omega)) \leq \sigma \mathfrak{Q}_{*}(\omega)+(1-\sigma) \mathfrak{Q}_{*}(\mathcal{Z}),
$$

and

$$
\mathfrak{Q}^{*}(\omega+(1-\sigma) \varphi(\mathcal{Z}, \omega)) \leq \sigma \mathfrak{Q}^{*}(\omega)+(1-\sigma) \mathfrak{Q}^{*}(\mathcal{Z}) .
$$

Hence, the result follows.

\section{Interval Fractional Hermite-Hadamard Inequalities}

In this section, we will prove two types of inequalities. First one is Hermite-Hadamard and their variant forms, and the second one is Hermite-Hadamard Fejér inequalities for LR-preinvex $I-V-F s$ where the integrands are $I-V-F s$. The family of Lebesgue measurable $I-V-F s$ is denoted by $L\left([t, t+\varphi(s, t)], \mathbb{R}_{I}^{+}\right)$in the following.

We need the following assumption regarding the function $\varphi:[s, t] \times[s, t] \rightarrow \mathbb{R}$, which plays an important role in upcoming main results.

Condition C. [44]

$$
\begin{gathered}
\varphi(\mathcal{Z}, \omega+\sigma \varphi(\mathcal{Z}, \omega))=(1-\sigma) \varphi(\mathcal{Z}, \omega) \\
\varphi(\omega, \omega+\sigma \varphi(\mathcal{Z}, \omega))=-\sigma \varphi(\mathcal{Z}, \omega) .
\end{gathered}
$$

Note that $\forall \omega, \mathcal{Z} \in[s, t]$ and $\varsigma \in[0,1]$, then from Condition $C$ we have

$$
\varphi\left(\omega+\sigma_{2} \varphi(\mathcal{Z}, \omega), \omega+\sigma_{1} \varphi(\mathcal{Z}, \omega)\right)=\left(\sigma_{2}-\sigma_{1}\right) \varphi(\mathcal{Z}, \omega) .
$$

Clearly for $\sigma=0$, we have $\xi(\mathcal{Z}, \omega)=0$ if and only if $\mathcal{Z}=\omega$, for all $\omega, \mathcal{Z} \in[s, t]$. For the application of Condition $C$, see $[28,34]$.

Theorem 3. Let $\mathfrak{Q}:[s, s+\varphi(t, s)] \rightarrow \mathbb{R}_{I}^{+}$be a LR-preinvex $I-V$-F on $[s, s+\varphi(t, s)]$ and given by $\mathfrak{Q}(\omega)=\left[\mathfrak{Q}_{*}(\omega), \mathfrak{Q}^{*}(\omega)\right]$ for all $\omega \in[s, s+\varphi(t, s)]$. If $\varphi$ satisfies Condition $C$ and $\mathfrak{Q} \in L\left([s, s+\varphi(t, s)], \mathbb{R}_{I}^{+}\right)$, then

$$
\begin{aligned}
\mathfrak{Q}\left(\frac{2 s+\varphi(t, s)}{2}\right) & \leq_{p} \frac{\Gamma(\alpha+1)}{2(\varphi(t, s))^{\alpha}}\left[\mathcal{I}_{s^{+}}^{\alpha} \mathfrak{Q}(s+\varphi(t, s))+\mathcal{I}_{s+\varphi(t, s)^{-}}^{\alpha} \mathfrak{Q}(s)\right] \\
& \leq_{p} \frac{\mathfrak{Q}(s)+\mathfrak{Q}(s+\varphi(t, s))}{2} \leq_{p} \frac{\mathfrak{Q}(s)+\mathfrak{Q}(t)}{2}
\end{aligned}
$$


If $\mathfrak{Q}(\omega)$ is $L R$ - preincave I- $V$-F then

$$
\begin{aligned}
\mathfrak{Q}\left(\frac{2 s+\varphi(t, s)}{2}\right) & \geq_{p} \frac{\Gamma(\alpha+1)}{2(\varphi(t, s))^{\alpha}}\left[\mathcal{I}_{s^{+}}^{\alpha} \mathfrak{Q}(s+\varphi(t, s))+\mathcal{I}_{s+\varphi(t, s)^{\alpha}}^{\alpha} \mathfrak{Q}(s)\right] \\
& \geq_{p} \frac{\mathfrak{Q}(s)+\mathfrak{Q}(s+\varphi(t, s))}{2} \geq_{p} \frac{\mathfrak{Q}(s)+\mathfrak{Q}(t)}{2} .
\end{aligned}
$$

Proof. Let $\mathfrak{Q}:[s, s+\varphi(t, s)] \rightarrow \mathbb{R}_{I}^{+}$be a LR-preinvex $I-V-F$. Then, by hypothesis, we have

$$
2 \mathfrak{Q}\left(\frac{2 s+\varphi(t, s)}{2}\right) \leq_{p} \mathfrak{Q}(s+(1-\sigma) \varphi(t, s))+\mathfrak{Q}(s+\sigma \varphi(t, s)) .
$$

Therefore, we have

$$
\begin{aligned}
2 \mathfrak{Q}_{*}\left(\frac{2 s+\varphi(t, s)}{2}\right) & \leq \mathfrak{Q}_{*}(s+(1-\sigma) \varphi(t, s))+\mathfrak{Q}_{*}(s+\sigma \varphi(t, s)), \\
2 \mathfrak{Q}^{*}\left(\frac{2 s+\varphi(t, s)}{2}\right) & \leq \mathfrak{Q}^{*}(s+(1-\sigma) \varphi(t, s))+\mathfrak{Q}^{*}(s+\sigma \varphi(t, s)) .
\end{aligned}
$$

Multiplying both sides by $\sigma^{\alpha-1}$ and integrating the obtained result with respect to $\sigma$ over $(0,1)$, we have

$$
\begin{aligned}
& 2 \int_{0}^{1} \sigma^{\alpha-1} \mathfrak{Q}_{*}\left(\frac{2 s+\varphi(t, s)}{2}\right) d \sigma \\
& \leq \int_{0}^{1} \sigma^{\alpha-1} \mathfrak{Q}_{*}(s+(1-\sigma) \varphi(t, s)) d \sigma+\int_{0}^{1} \sigma^{\alpha-1} \mathfrak{Q}_{*}(s+\sigma \varphi(t, s)) d \sigma, \\
& 2 \int_{0}^{1} \sigma^{\alpha-1} \mathfrak{Q}^{*}\left(\frac{2 s+\varphi(t, s)}{2}\right) d \sigma \\
& \leq \int_{0}^{1} \sigma^{\alpha-1} \mathfrak{Q}^{*}(s+(1-\sigma) \varphi(t, s)) d \sigma+\int_{0}^{1} \sigma^{\alpha-1} \mathfrak{Q}^{*}(s+\sigma \varphi(t, s)) d \sigma .
\end{aligned}
$$

Let $\omega=s+(1-\sigma) \varphi(t, s)$ and $\mathcal{Z}=s+\sigma \varphi(t, s)$. Then we have

$$
\begin{gathered}
\frac{2}{\alpha} \mathfrak{Q}_{*}\left(\frac{2 s+\varphi(t, s)}{2}\right) \leq \frac{1}{(\varphi(t, s))^{\alpha}} \int_{s}^{s+\varphi(t, s)}(s+\varphi(t, s)-\mathcal{Z})^{\alpha-1} \mathfrak{Q}_{*}(\mathcal{Z}) d \mathcal{Z} \\
\quad+\frac{1}{(\varphi(t, s))^{\alpha}} \int_{s}^{s+\varphi(t, s)}(\omega-s)^{\alpha-1} \mathfrak{Q}_{*}(\omega) d \omega \\
\frac{2}{\alpha} \mathfrak{Q}_{*}\left(\frac{2 s+\varphi(t, s)}{2}\right) \leq \frac{1}{(\varphi(t, s))^{\alpha}} \int_{s}^{s+\varphi(t, s)}(s+\varphi(t, s)-\mathcal{Z})^{\alpha-1} \mathfrak{Q}^{*}(\mathcal{Z}) d \mathcal{Z} \\
\quad+\frac{1}{(\varphi(t, s))^{\alpha}} \int_{s}^{s+\varphi(t, s)}(\omega-s)^{\alpha-1} \mathfrak{Q}^{*}(\omega) d \omega, \\
\leq \frac{\Gamma(\alpha)}{(\varphi(t, s))^{\alpha}}\left[\mathcal{I}_{s^{+}}^{\alpha} \mathfrak{Q}_{*}(s+\varphi(t, s))+\mathcal{I}_{s+\varphi(t, s)^{-}}^{\alpha} \mathfrak{Q}_{*}(s)\right] \\
\leq \frac{\Gamma(\alpha)}{(\varphi(t, s))^{\alpha}}\left[\mathcal{I}_{s^{+}}^{\alpha} \mathfrak{Q}^{*}(s+\varphi(t, s))+\mathcal{I}_{s+\varphi(t, s)^{-}}^{\alpha} \mathfrak{Q}^{*}(s)\right],
\end{gathered}
$$

That is

$$
\begin{gathered}
\frac{2}{\alpha}\left[\mathfrak{Q}_{*}\left(\frac{2 s+\varphi(t, s)}{2}\right), \mathfrak{Q}^{*}\left(\frac{2 s+\varphi(t, s)}{2}\right)\right] \\
\leq_{p} \frac{\Gamma(\alpha)}{(\varphi(t, s))^{\alpha}}\left[\left[\begin{array}{c}
\mathcal{I}_{s^{+}}^{\alpha} \mathfrak{Q}_{*}(s+\varphi(t, s)) \\
+\mathcal{I}_{s+\varphi(t, s)^{-}}^{\alpha} \mathfrak{Q}_{*}(s)
\end{array}\right],\left[\begin{array}{c}
\mathcal{I}_{s^{+}}^{\alpha} \mathfrak{Q}^{*}(s+\varphi(t, s)) \\
+\mathcal{I}_{t^{-}}^{\alpha} \mathfrak{Q}^{*}(s+\varphi(t, s))
\end{array}\right]\right] .
\end{gathered}
$$

Thus,

$$
\frac{2}{\alpha} \mathfrak{Q}\left(\frac{2 s+\varphi(t, s)}{2}\right) \leq_{p} \frac{\Gamma(\alpha)}{(\varphi(t, s))^{\alpha}}\left[\mathcal{I}_{s^{+}}^{\alpha} \mathfrak{Q}(s+\varphi(t, s))+\mathcal{I}_{s+\varphi(t, s)^{-}}^{\alpha} \mathfrak{Q}(s)\right] .
$$

In a similar way as above, we have

$$
\frac{\Gamma(\alpha)}{(\varphi(t, s))^{\alpha}}\left[\mathcal{I}_{s^{+}}^{\alpha} \mathfrak{Q}(s+\varphi(t, s))+\mathcal{I}_{s+\varphi(t, s)^{-}}^{\alpha} \mathfrak{Q}(s)\right] \leq_{p} \frac{\mathfrak{Q}(s)+\mathfrak{Q}(s+\varphi(t, s))}{2} \leq_{p} \frac{\mathfrak{Q}(s)+\mathfrak{Q}(t)}{2} .
$$


Combining (8) and (9), we have

$$
\begin{aligned}
\mathfrak{Q}\left(\frac{2 s+\varphi(t, s)}{2}\right) & \leq p \frac{\Gamma(\alpha+1)}{2(\varphi(t, s))^{\alpha}}\left[\mathcal{I}_{s^{+}}^{\alpha} \mathfrak{Q}(s+\varphi(t, s))+\mathcal{I}_{s+\varphi(t, s)^{\alpha}}^{\alpha} \mathfrak{Q}(s)\right] \\
& \leq_{p} \frac{\mathfrak{Q}(s)+\mathfrak{Q}(s+\varphi(t, s))}{2} \leq_{p} \frac{\mathfrak{Q}(s)+\mathfrak{Q}(t)}{2} .
\end{aligned}
$$

Hence, the required result.

Remark 2. From Theorem 3 we clearly see that

If $\varphi(\omega, \mathcal{Z})=\omega-\mathcal{Z}$, then from Theorem 3, we obtain the following new result in fractional calculus, see [43].

$$
\mathfrak{Q}\left(\frac{s+t}{2}\right) \leq_{p} \frac{\Gamma(\alpha+1)}{2(t-s)^{\alpha}}\left[\mathcal{I}_{s^{+}}^{\alpha} \mathfrak{Q}(t)+\mathcal{I}_{t^{-}}^{\alpha} \mathfrak{Q}(s)\right] \leq_{p} \frac{\mathfrak{Q}(s)+\mathfrak{Q}(t)}{2} .
$$

Let $\alpha=1$. Then Theorem 3 reduces to the result for LR-preinvex I-V-F given in [44]:

$$
\mathfrak{Q}\left(\frac{2 s+\varphi(t, s)}{2}\right) \leq_{p} \frac{1}{\varphi(t, s)} \int_{s}^{s+\varphi(t, s)} \mathfrak{Q}(\omega) d \omega \leq_{p} \frac{\mathfrak{Q}(s)+\mathfrak{Q}(t)}{2}
$$

Let $\alpha=1$ and $\varphi(\omega, \mathcal{Z})=\omega-\mathcal{Z}$. Then Theorem 3 reduces to the result for convex $I-V-F$ given in [44]:

$$
\mathfrak{Q}\left(\frac{s+t}{2}\right) \leq_{p} \frac{1}{t-s} \int_{s}^{t} \mathfrak{Q}(\omega) d \omega \leq_{p} \frac{\mathfrak{Q}(s)+\mathfrak{Q}(t)}{2}
$$

Let $\alpha=1$ and $\mathfrak{Q}_{*}(\omega)=\mathfrak{Q}^{*}(\omega)$ with $\varphi(\omega, \mathcal{Z})=\omega-\mathcal{Z}$. Then from Theorem 3 we obtain classical Hermite-Hadamard Fejértype inequality.

Example 1. Let $\alpha=\frac{1}{2}, \omega \in[2,2+\varphi(3,2)]$, and the $I-V-F \mathfrak{Q}:[s, s+\varphi(t, s)]=[2,2+\varphi(3,2)]$ $\rightarrow \mathbb{R}_{I}^{+}$, such that $\mathfrak{Q}(\omega)=[1,2]\left(2-\omega^{\frac{1}{2}}\right)$. Since left and right end point functions $\mathfrak{Q}_{*}(\omega)=2-\omega^{\frac{1}{2}}, \mathfrak{Q}^{*}(\omega)=2\left(2-\omega^{\frac{1}{2}}\right)$, are LR-preinvex functions with respect to $\varphi(t, s)=t-s$, then $\mathfrak{Q}(\omega)$ is $L R$-preinvex I-V-F. We clearly see that $\mathfrak{Q} \in L\left([s, s+\varphi(t, s)], \mathbb{R}_{I}^{+}\right)$and

$$
\begin{gathered}
\mathfrak{Q}_{*}\left(\frac{2 s+\varphi(t, s)}{2}\right)=\mathfrak{Q}_{*}\left(\frac{5}{2}\right)=\frac{4-\sqrt{10}}{2} \\
\mathfrak{Q}^{*}\left(\frac{2 s+\varphi(t, s)}{2}\right)=\mathfrak{Q}^{*}\left(\frac{5}{2}\right)=4-\sqrt{10}, \\
\frac{\mathfrak{Q}_{*}(s)+\mathfrak{Q}_{*}(s+\varphi(t, s))}{2}=\frac{4-\sqrt{2}-\sqrt{3}}{2} \\
\frac{\mathfrak{Q}^{*}(s)+\mathfrak{Q}^{*}(s+\varphi(t, s))}{2}=4-\sqrt{2}-\sqrt{3} .
\end{gathered}
$$

Note that

$$
\begin{gathered}
\frac{\Gamma(\alpha+1)}{2(\varphi(t, s))^{\alpha}}\left[\mathcal{I}_{s^{+}}^{\alpha} \mathfrak{Q}_{*}(s+\varphi(t, s))+\mathcal{I}_{s+\varphi(t, s)^{-}}^{\alpha} \mathfrak{Q}_{*}(s)\right] \\
=\frac{\Gamma\left(\frac{3}{2}\right)}{2} \frac{1}{\sqrt{\pi}} \int_{2}^{2+\varphi(3,2)}(3-\omega)^{\frac{-1}{2}} \cdot\left(2-\omega^{\frac{1}{2}}\right) d \omega \\
+\frac{\Gamma\left(\frac{3}{2}\right)}{2} \frac{1}{\sqrt{\pi}} \int_{2}^{2+\varphi(3,2)}(\omega-2)^{\frac{-1}{2}} \cdot\left(2-\omega^{\frac{1}{2}}\right) d \omega \\
=\frac{1}{4}\left[\frac{7393}{10,000}+\frac{9501}{10,000}\right] \\
=\frac{8447}{20,000} . \\
\frac{\Gamma(\alpha+1)}{2(\varphi(t, s))^{\alpha}}\left[\mathcal{I}_{s^{+}}^{\alpha} \mathfrak{Q}^{*}(s+\varphi(t, s))+\mathcal{I}_{s+\varphi(t, s)^{-}}^{\alpha} \mathfrak{Q}^{*}(s)\right] \\
=\frac{\Gamma\left(\frac{3}{2}\right)}{2} \frac{1}{\sqrt{\pi}} \int_{2}^{2+\varphi(3,2)}(3-\omega)^{\frac{-1}{2}} \cdot 2\left(2-\omega^{\frac{1}{2}}\right) d \omega \\
+\frac{\Gamma\left(\frac{3}{2}\right)}{2} \frac{1}{\sqrt{\pi}} \int_{2}^{2+\varphi(3,2)}(\omega-2)^{\frac{-1}{2}} \cdot 2\left(2-\omega^{\frac{1}{2}}\right) d \omega \\
=\frac{1}{2}\left[\frac{7393}{10,000}+\frac{9501}{10,000}\right]=\frac{8447}{10,000} .
\end{gathered}
$$


Therefore,

$$
\left[\frac{4-\sqrt{10}}{2}, 4-\sqrt{10}\right] \leq_{p}\left[\frac{8447}{20,000}, \frac{8447}{10,000}\right] \leq_{p}\left[\frac{4-\sqrt{2}-\sqrt{3}}{2}, 4-\sqrt{2}-\sqrt{3}\right],
$$

and Theorem 3 is verified.

It is well known fact that Hermite-Hadamard Fejér type inequality is a generalization of Hermite-Hadamard type inequality. In Theorem 4 and Theorem 5, we obtain second and first fractional Hermite-Hadamard Fejér type inequalities for introduced LR-preinvex $I-V-F$, respectively.

Theorem 4. Let $\mathfrak{Q}:[s, s+\varphi(t, s)] \rightarrow \mathbb{R}_{I}^{+}$be a LR-preinvex $I-V-F$ with $s<s+\varphi(t, s)$, and given by $\mathfrak{Q}(\omega)=\left[\mathfrak{Q}_{*}(\omega), \mathfrak{Q}^{*}(\omega)\right]$ for all $\omega \in[s, s+\varphi(t, s)]$. Let $\mathfrak{Q} \in L\left([s, s+\varphi(t, s)], \mathbb{R}_{I}^{+}\right)$ and $\mathcal{D}:[s, s+\varphi(t, s)] \rightarrow \mathbb{R}, \mathcal{D}(\omega) \geq 0$, symmetric with respect to $\frac{2 s+\varphi(t, s)}{2}$. If $\varphi$ satisfies Condition $C$ and then

$$
\begin{gathered}
{\left[\mathcal{I}_{s^{+}}^{\alpha} \mathfrak{Q D}(s+\varphi(t, s))+\mathcal{I}_{s+\varphi(t, s)^{-}}^{\alpha} \mathfrak{Q D}(s)\right] \leq_{p} \frac{\mathfrak{Q}(s)+\mathfrak{Q}(s+\varphi(t, s))}{2}\left[\begin{array}{c}
\mathcal{I}_{s^{+}}^{\alpha} D(s+\varphi(t, s)) \\
+\mathcal{I}_{s+\varphi(t, s)^{-}}^{\alpha} D(s)
\end{array}\right] \leq_{p} \frac{\mathfrak{Q}(s)+\mathfrak{Q}(t)}{2}} \\
{\left[\mathcal{I}_{s^{+}}^{\alpha} \mathcal{D}(s+\varphi(t, s))+\mathcal{I}_{s+\varphi(t, s)^{-}}^{\alpha} \mathcal{D}(s)\right]}
\end{gathered}
$$

If $\mathfrak{Q}$ is preincave $I-V-F$, then inequality (10) is reversed.

Proof. Let $\mathfrak{Q}$ be a LR-preinvex $I-V-F$ and $\sigma^{\alpha-1} \mathcal{D}(s+(1-\sigma) \varphi(t, s)) \geq 0$. Then, we have

$$
\begin{aligned}
\sigma^{\alpha-1} & \mathfrak{Q}_{*}(s+(1-\sigma) \varphi(t, s)) \mathcal{D}(s+(1-\sigma) \varphi(t, s)) \\
& \leq \sigma^{\alpha-1}\left(\sigma \mathfrak{Q}_{*}(s)+(1-\sigma) \mathfrak{Q}_{*}(s+\varphi(t, s))\right) \mathcal{D}(s+(1-\sigma) \varphi(s+\varphi(t, s), s)) \\
\sigma^{\alpha-1} & \mathfrak{Q}^{*}(s+(1-\sigma) \varphi(t, s)) \mathcal{D}(s+(1-\sigma) \varphi(t, s)) \\
& \leq \sigma^{\alpha-1}\left(\sigma \mathfrak{Q}^{*}(s)+(1-\sigma) \mathfrak{Q}^{*}(s+\varphi(t, s))\right) \mathcal{D}(s+(1-\sigma) \varphi(s+\varphi(t, s), s)) .
\end{aligned}
$$

Plus,

$$
\begin{aligned}
\sigma^{\alpha-1} & \mathfrak{Q}_{*}(s+\sigma \varphi(t, s)) \mathcal{D}(s+\sigma \varphi(t, s)) \\
& \leq \sigma^{\alpha-1}\left((1-\sigma) \mathfrak{Q}_{*}(s)+\sigma \mathfrak{Q}_{*}(s+\varphi(t, s))\right) \mathcal{D}(s+\sigma \varphi(t, s)) \\
\sigma^{\alpha-1} & \mathfrak{Q}^{*}(s+\sigma \varphi(t, s)) \mathcal{D}(s+\sigma \varphi(t, s)) \\
& \leq \sigma^{\alpha-1}\left((1-\sigma) \mathfrak{Q}^{*}(s)+\sigma \mathfrak{Q}^{*}(s+\varphi(t, s))\right) \mathcal{D}(s+\sigma \varphi(t, s)) .
\end{aligned}
$$

After adding (11) and (12), and integrating over [0, 1], we obtain

$$
\begin{aligned}
& \int_{0}^{1} \sigma^{\alpha-1} \mathfrak{Q}_{*}(s+(1-\sigma) \varphi(t, s)) \mathcal{D}(s+(1-\sigma) \varphi(t, s)) d \sigma \\
& +\int_{0}^{1} \sigma^{\alpha-1} \mathfrak{Q}_{*}(s+\sigma \varphi(t, s)) \mathcal{D}(s+\sigma \varphi(t, s)) d \sigma \\
& \leq \int_{0}^{1}\left[\begin{array}{c}
\sigma^{\alpha-1} \mathfrak{Q}_{*}(s)\{\sigma \mathcal{D}(s+(1-\sigma) \varphi(t, s))+(1-\sigma) \mathcal{D}(s+\sigma \varphi(t, s))\} \\
+\sigma^{\alpha-1} \mathfrak{Q}_{*}(s+\varphi(t, s))\{(1-\sigma) \mathcal{D}(s+(1-\sigma) \varphi(t, s))+\sigma \mathcal{D}(s+\sigma \varphi(t, s))\}
\end{array}\right] d \sigma, \\
& \int_{0}^{1} \sigma^{\alpha-1} \mathfrak{Q}^{*}(s+\sigma \varphi(t, s)) \mathcal{D}(s+\sigma \varphi(t, s)) d \sigma \\
& +\int_{0}^{1} \sigma^{\alpha-1} \mathfrak{Q}^{*}(s+(1-\sigma) \varphi(t, s)) \mathcal{D}(s+(1-\sigma) \varphi(t, s)) d \sigma \\
& \leq \int_{0}^{1}\left[\begin{array}{c}
\sigma^{\alpha-1} \mathfrak{Q}^{*}(s)\{\sigma \mathcal{D}(s+(1-\sigma) \varphi(t, s))+(1-\sigma) \mathcal{D}(s+\sigma \varphi(t, s))\} \\
+\sigma^{\alpha-1} \mathfrak{Q}^{*}(s+\varphi(t, s))\{(1-\sigma) \mathcal{D}(s+(1-\sigma) \varphi(t, s))+\sigma \mathcal{D}(s+\sigma \varphi(t, s))\}
\end{array}\right] d \sigma, \\
& =\mathfrak{Q}_{*}(s) \int_{0}^{1} \sigma^{\alpha-1} D(s+(1-\sigma) \varphi(t, s)) d \sigma+\mathfrak{Q}_{*}(s+\varphi(t, s)) \int_{0}^{1} \sigma^{\alpha-1} D(s+\sigma \varphi(t, s)) d \sigma \text {, } \\
& =\mathfrak{Q}^{*}(s) \int_{0}^{1} \sigma^{\alpha-1} D(s+(1-\sigma) \varphi(t, s)) d \sigma+\mathfrak{Q}^{*}(s+\varphi(t, s)) \int_{0}^{1} \sigma^{\alpha-1} D(s+\sigma \varphi(t, s)) d \sigma \text {. }
\end{aligned}
$$


Since $\mathcal{D}$ is symmetric, then

$$
\begin{array}{r}
=\left[\mathfrak{Q}_{*}(s)+\mathfrak{Q}_{*}(s+\varphi(t, s))\right] \int_{0}^{1} \sigma^{\alpha-1} D(s+\sigma \varphi(t, s)) d \sigma \\
=\left[\mathfrak{Q}^{*}(s)+\mathfrak{Q}^{*}(s+\varphi(t, s))\right] \int_{0}^{1} \sigma^{\alpha-1} D(s+\sigma \varphi(t, s)) d \sigma . \\
=\frac{\mathfrak{Q}_{*}(s)+\mathfrak{Q}_{*}(s+\varphi(t, s))}{2} \frac{\Gamma(\alpha)}{(\varphi(t, s))^{\alpha}}\left[\mathcal{I}_{s^{+}}^{\alpha} \mathcal{D}(s+\varphi(t, s))+\mathcal{I}_{s+\varphi(t, s)}^{\alpha} \mathcal{D}(s)\right], \\
=\frac{\mathfrak{Q}^{*}(s)+\mathfrak{Q}^{*}(s+\varphi(t, s))}{2} \frac{\Gamma(\alpha)}{(\varphi(t, s))^{\alpha}}\left[\mathcal{I}_{s^{+}}^{\alpha} \mathcal{D}(s+\varphi(t, s))+\mathcal{I}_{s+\varphi(t, s)^{\alpha}}^{\alpha} \mathcal{D}(s)\right] .
\end{array}
$$

Since

$$
\begin{aligned}
\int_{0}^{1} \sigma^{\alpha-1} \mathfrak{Q}_{*}(s+ & (1-\sigma) \varphi(t, s)) \mathcal{D}(s+\sigma \varphi(t, s)) d \sigma \\
= & \frac{1}{(\varphi(t, s))^{\alpha}} \int_{s}^{s+\varphi(t, s)}(\omega-s)^{\alpha-1} \mathfrak{Q}_{*}(2 s+\varphi(t, s)-\omega) \mathcal{D}(\omega) d \omega \\
& +\frac{1}{(\varphi(t, s))^{\alpha}} \int_{s}^{s+\varphi(t, s)}(\omega-s)^{\alpha-1} \mathfrak{Q}_{*}(\omega) \mathcal{D}(\omega) d \omega \\
= & \frac{1}{(\varphi(t, s))^{\alpha}} \int_{s}^{s+\varphi(t, s)}(\omega-s)^{\alpha-1} \mathfrak{Q}_{*}(\omega) \mathcal{D}(2 s+\varphi(t, s)-\omega) d \omega \\
& +\frac{1}{(\varphi(t, s))^{\alpha}} \int_{s}^{s+\varphi(t, s)}(\omega-s)^{\alpha-1} \mathfrak{Q}_{*}(\omega) \mathcal{D}(\omega) d \omega \\
= & \frac{\Gamma(\alpha)}{(\varphi(t, s))^{\alpha}}\left[\mathcal{I}_{s^{+}}^{\alpha} \mathfrak{Q}_{*} \mathcal{D}(t)+\mathcal{I}_{t^{-}}^{\alpha} \mathfrak{Q}_{*} \mathcal{D}(s)\right], \\
\int_{0}^{1} \sigma^{\alpha-1} \mathfrak{Q}^{*}(s+ & (1-\sigma) \varphi(t, s)) \mathcal{D}(s+\sigma \varphi(t, s)) d \sigma \\
& \quad+\int_{0}^{1} \sigma^{\alpha-1} \mathfrak{Q}^{*}(s+\sigma \varphi(t, s)) \mathcal{D}(s+\sigma \varphi(t, s)) d \sigma \\
= & \frac{\Gamma(\alpha)}{(\varphi(t, s))^{\alpha}}\left[\mathcal{I}_{s^{+}}^{\alpha} \mathfrak{Q}^{*} \mathcal{D}(s+\varphi(t, s))+\mathcal{I}_{s+\varphi(t, s)^{-}}^{\alpha} \mathfrak{Q}^{*} \mathcal{D}(s)\right] .
\end{aligned}
$$

Then from (13), we have

$$
\begin{aligned}
\frac{\Gamma(\alpha)}{(\varphi(t, s))^{\alpha}} & {\left[\mathcal{I}_{s^{+}}^{\alpha} \mathfrak{Q}_{*} \mathcal{D}(s+\varphi(t, s))+\mathcal{I}_{s+\varphi(t, s)^{-}}^{\alpha} \mathfrak{Q}_{*} \mathcal{D}(s)\right] } \\
& \leq \frac{\mathfrak{Q}_{*}(s)+\mathfrak{Q}_{*}(s+\varphi(t, s))}{2} \frac{\Gamma(\alpha)}{(\varphi(t, s))^{\alpha}}\left[\mathcal{I}_{s^{+}}^{\alpha} \mathcal{D}(s+\varphi(t, s))+\mathcal{I}_{s+\varphi(t, s)^{-}}^{\alpha} \mathcal{D}(s)\right] \\
& \leq \frac{\mathfrak{Q}_{*}(s)+\mathfrak{Q}_{*}(s+\varphi(t, s))}{2} \frac{\Gamma(\alpha)}{(\varphi(t, s))^{\alpha}}\left[\mathcal{I}_{s^{+}}^{\alpha} \mathcal{D}(s+\varphi(t, s))+\mathcal{I}_{s+\varphi(t, s)^{-}}^{\alpha} \mathcal{D}(s)\right], \\
\frac{\Gamma(\alpha)}{(\varphi(t, s))^{\alpha}} & {\left[\mathcal{I}_{s^{+}}^{\alpha} \mathfrak{Q}^{*} \mathcal{D}(s+\varphi(t, s))+\mathcal{I}_{s+\varphi(t, s)^{-}}^{\alpha} \mathfrak{Q}^{*} \mathcal{D}(s)\right] } \\
& \leq \frac{\mathfrak{Q}^{*}(s)+\mathfrak{Q}^{*}(s+\varphi(t, s))}{2} \frac{\Gamma(\alpha)}{(\varphi(t, s))^{\alpha}}\left[\mathcal{I}_{s^{+}}^{\alpha} \mathcal{D}(s+\varphi(t, s))+\mathcal{I}_{s+\varphi(t, s)^{-}}^{\alpha} \mathcal{D}(s)\right] \\
& \leq \frac{\mathfrak{Q}^{*}(s)+\mathfrak{Q}^{*}(s+\varphi(t))}{2} \frac{\Gamma(\alpha)}{(\varphi(t, s))^{\alpha}}\left[\mathcal{I}_{s^{+}}^{\alpha} \mathcal{D}(s+\varphi(t, s))+\mathcal{I}_{s+\varphi(t, s)^{-}}^{\alpha} \mathcal{D}(s)\right],
\end{aligned}
$$

that is

$$
\begin{aligned}
& \frac{\Gamma(\alpha)}{(\varphi(t, s))^{\alpha}}\left[\mathcal{I}_{s^{+}}^{\alpha} \mathfrak{Q}_{*} \mathcal{D}(s+\varphi(t, s))+\mathcal{I}_{s+\varphi(t, s)^{-}}^{\alpha} \mathfrak{Q}_{*} \mathcal{D}(s), \mathcal{I}_{s^{+}}^{\alpha} \mathfrak{Q}^{*} \mathcal{D}(s+\varphi(t, s))+\mathcal{I}_{s+\varphi(t, s)^{-}}^{\alpha} \mathfrak{Q}^{*} \mathcal{D}(s)\right] \\
& \leq_{p} \frac{\Gamma(\alpha)}{(\varphi(t, s))^{\alpha}}\left[\frac{\mathfrak{Q}_{*}(s)+\mathfrak{Q}_{*}(s+\varphi(t, s))}{2}, \frac{\mathfrak{Q}^{*}(s)+\mathfrak{Q}^{*}(s+\varphi(t, s))}{2}\right]\left[\mathcal{I}_{s^{+}}^{\alpha} \mathcal{D}(s+\varphi(t, s))+\mathcal{I}_{s+\varphi(t, s)^{-}}^{\alpha} \mathcal{D}(s)\right] \\
& \leq_{p} \frac{\Gamma(\alpha)}{(\varphi(t, s))^{\alpha}}\left[\frac{\mathfrak{Q}_{*}(s)+\mathfrak{Q}_{*}(t)}{2}, \frac{\mathfrak{Q}^{*}(s)+\mathfrak{Q}^{*}(t)}{2}\right]\left[\mathcal{I}_{s^{+}}^{\alpha} \mathcal{D}(s+\varphi(t, s))+\mathcal{I}_{s+\varphi(t, s)^{-}}^{\alpha} \mathcal{D}(s)\right] \\
& {\left[\mathcal{I}_{s^{+}}^{\alpha} \mathfrak{Q D}(s+\varphi(t, s)) \leq_{p} \mathcal{I}_{s+\varphi(t, s)^{-}}^{\alpha} \mathfrak{Q D}(s)\right] \leq_{p} \frac{\mathfrak{Q}(s)+\mathfrak{Q}(s+\varphi(t, s))}{2}\left[\begin{array}{c}
\mathcal{I}_{s^{+}}^{\alpha} D(s+\varphi(t, s)) \\
+\mathcal{I}_{s+\varphi(t, s)^{-}}^{\alpha} D(s)
\end{array}\right] \leq_{p} \frac{\mathfrak{Q}(s)+\mathfrak{Q}(t)}{2}} \\
& {\left[\mathcal{I}_{s^{+}}^{\alpha} \mathcal{D}(s+\varphi(t, s))+\mathcal{I}_{s+\varphi(t, s)^{-}}^{\alpha} \mathcal{D}(s)\right]}
\end{aligned}
$$


Theorem 5. Let $\mathfrak{Q}:[s, s+\varphi(t, s)] \rightarrow \mathbb{R}_{I}^{+}$be a LR-preinvex $I-V-F$ with $s<s+\varphi(t, s)$, and defined by $\mathfrak{Q}(\omega)=\left[\mathfrak{Q}_{*}(\omega), \mathfrak{Q}^{*}(\omega)\right]$ for all $\omega \in[s, s+\varphi(t, s)]$. If $\mathfrak{Q} \in L\left([s, s+\varphi(t, s)], \mathbb{R}_{I}^{+}\right)$ and $\mathcal{D}:[s, s+\varphi(t, s)] \rightarrow \mathbb{R}, \mathcal{D}(\omega) \geq 0$, symmetric with respect to $\frac{2 s+\varphi(t, s)}{2}$. If $\varphi$ satisfies Condition $C$ and then

$\mathfrak{Q}\left(\frac{2 s+\varphi(t, s)}{2}\right)\left[\mathcal{I}_{s^{+}}^{\alpha} \mathcal{D}(s+\varphi(t, s))+\mathcal{I}_{s+\varphi(t, s)^{-}}^{\alpha} \mathcal{D}(s)\right] \leq_{p}\left[\mathcal{I}_{s^{+}}^{\alpha} \mathfrak{Q D}(s+\varphi(t, s))+\mathcal{I}_{s+\varphi(t, s)^{-}}^{\alpha} \mathfrak{Q D}(s)\right]$.

If $\mathfrak{Q}$ is preincave $I-V-F$, then inequality (15) is reversed.

Proof. Since $\mathfrak{Q}$ is a LR-preinvex $I-V-F$, then we have

$$
\begin{aligned}
& \mathfrak{Q}_{*}\left(\frac{2 s+\varphi(t, s)}{2}\right) \leq \frac{1}{2}\left(\mathfrak{Q}_{*}(s+(1-\sigma) \varphi(t, s))+\mathfrak{Q}_{*}(s+\sigma \varphi(t, s))\right) \\
& \mathfrak{Q}^{*}\left(\frac{2 s+\varphi(t, s)}{2}\right) \leq \frac{1}{2}\left(\mathfrak{Q}^{*}(s+(1-\sigma) \varphi(t, s))+\mathfrak{Q}^{*}(s+\sigma \varphi(t, s))\right),
\end{aligned}
$$

Since $\mathcal{D}(s+(1-\sigma) \varphi(t, s))=\mathcal{D}(s+\sigma \varphi(t, s))$, then by multiplying (16) by $\sigma^{\alpha-1} \mathcal{D}(s+\sigma \varphi(t, s))$ and integrate it with respect to $\sigma$ over $[0,1]$, we obtain

$$
\begin{aligned}
\mathfrak{Q}_{*}\left(\frac{2 s+\varphi(t, s)}{2}\right) & \int_{0}^{1} \sigma^{\alpha-1} \mathcal{D}(s+\sigma \varphi(t, s)) d \sigma \\
\leq & \frac{1}{2}\left(\begin{array}{c}
\int_{0}^{1} \sigma^{\alpha-1} \mathfrak{Q}_{*}(s+(1-\sigma) \varphi(t, s)) \mathcal{D}(s+\sigma \varphi(t, s)) d \sigma \\
+\int_{0}^{1} \sigma^{\alpha-1} \mathfrak{Q}_{*}(s+\sigma \varphi(t, s)) \mathcal{D}(s+\sigma \varphi(t, s)) d \sigma
\end{array}\right), \\
\mathfrak{Q}^{*}\left(\frac{2 s+\varphi(t, s)}{2}\right) & \int_{0}^{1} \mathcal{D}(s+\sigma \varphi(t, s)) d \sigma \\
\leq & \frac{1}{2}\left(\begin{array}{c}
\int_{0}^{1} \sigma^{\alpha-1} \mathfrak{Q}^{*}(s+(1-\sigma) \varphi(t, s)) \mathcal{D}(s+\sigma \varphi(t, s)) d \sigma \\
+\int_{0}^{1} \sigma^{\alpha-1} \mathfrak{Q}^{*}(s+\sigma \varphi(t, s)) \mathcal{D}(s+\sigma \varphi(t, s)) d \sigma
\end{array}\right) .
\end{aligned}
$$

Let $\omega=s+\sigma \varphi(t, s)$. Then, we have

$$
\begin{aligned}
\int_{0}^{1} \sigma^{\alpha-1} \mathfrak{Q}_{*}(s+ & (1-\sigma) \varphi(t, s)) \mathcal{D}(s+\sigma \varphi(t, s)) d \sigma \\
& +\int_{0}^{1} \sigma^{\alpha-1} \mathfrak{Q}_{*}(s+\sigma \varphi(t, s)) \mathcal{D}(s+\sigma \varphi(t, s)) d \sigma \\
= & \frac{1}{(\varphi(t, s))^{\alpha}} \int_{s}^{s+\varphi(t, s)}(\omega-s)^{\alpha-1} \mathfrak{Q}_{*}(2 s+\varphi(t, s)-\omega) \mathcal{D}(\omega) d \omega \\
& +\frac{1}{(\varphi(t, s))^{\alpha}} \int_{s}^{s+\varphi(t, s)}(\omega-s)^{\alpha-1} \mathfrak{Q}_{*}(\omega) \mathcal{D}(\omega) d \omega \\
= & \frac{1}{(\varphi(t, s))^{\alpha}} \int_{s}^{s+\varphi(t, s)}(\omega-s)^{\alpha-1} \mathfrak{Q}_{*}(\omega) \mathcal{D}(2 s+\varphi(t, s)-\omega) d \omega \\
& +\frac{1}{(\varphi(t, s))^{\alpha}} \int_{s}^{s+\varphi(t, s)}(\omega-s)^{\alpha-1} \mathfrak{Q}_{*}(\omega) \mathcal{D}(\omega) d \omega \\
= & \frac{\Gamma(\alpha)}{(\varphi(t, s))^{\alpha}}\left[\mathcal{I}_{s^{+}}^{\alpha} \mathfrak{Q}_{*} \mathcal{D}(s+\varphi(t, s))+\mathcal{I}_{s+\varphi(t, s)^{-}}^{\alpha} \mathfrak{Q}_{*} \mathcal{D}(s)\right], \\
\int_{0}^{1} \sigma^{\alpha-1} \mathfrak{Q}^{*}(s+(1-\sigma) \varphi(t, s)) \mathcal{D}(s+\sigma \varphi(t, s)) d \sigma & \quad+\int_{0}^{1} \sigma^{\alpha-1} \mathfrak{Q}^{*}(s+\sigma \varphi(t, s)) \mathcal{D}(s+\sigma \varphi(t, s)) d \sigma \\
= & \frac{\Gamma(\alpha)}{(\varphi(t, s))^{\alpha}}\left[\mathcal{I}_{s^{+}}^{\alpha} \mathfrak{Q}^{*} \mathcal{D}(s+\varphi(t, s))+\mathcal{I}_{s+\varphi(t, s)^{-}}^{\alpha} \mathfrak{Q}^{*} \mathcal{D}(s)\right] .
\end{aligned}
$$

Then from (18), we have

$$
\begin{array}{r}
\frac{\Gamma(\alpha)}{(\varphi(t, s))^{\alpha}} \mathfrak{Q}_{*}\left(\frac{2 s+\varphi(t, s)}{2}\right)\left[\mathcal{I}_{s^{+}}^{\alpha} \mathcal{D}(s+\varphi(t, s))+\mathcal{I}_{s+\varphi(t, s)^{-}}^{\alpha} \mathcal{D}(s)\right] \\
\leq \frac{\Gamma(\alpha)}{(\varphi(t, s))^{\alpha}}\left[\mathcal{I}_{s^{+}}^{\alpha} \mathfrak{Q}_{*} \mathcal{D}(s+\varphi(t, s))+\mathcal{I}_{s+\varphi(t, s)^{-}}^{\alpha} \mathfrak{Q}_{*} \mathcal{D}(s)\right] \\
\frac{\Gamma(\alpha)}{(\varphi(t, s))^{\alpha}} \mathfrak{Q}^{*}\left(\frac{2 s+\varphi(t, s)}{2}\right)\left[\mathcal{I}_{s^{+}}^{\alpha} \mathcal{D}(s+\varphi(t, s))+\mathcal{I}_{s+\varphi(t, s)^{-}}^{\alpha} \mathcal{D}(s)\right] \\
\leq \frac{\Gamma(\alpha)}{(\varphi(t, s))^{\alpha}}\left[\mathcal{I}_{s^{+}}^{\alpha} \mathfrak{Q}^{*} \mathcal{D}(s+\varphi(t, s))+\mathcal{I}_{s+\varphi(t, s)^{-}}^{\alpha} \mathfrak{Q}^{*} \mathcal{D}(s)\right],
\end{array}
$$


from which, we have

$$
\begin{aligned}
\frac{\Gamma(\alpha)}{(\varphi(t, s))^{\alpha}} & {\left[\mathfrak{Q}_{*}\left(\frac{2 s+\varphi(t, s)}{2}\right), \mathfrak{Q}^{*}\left(\frac{2 s+\varphi(t, s)}{2}\right)\right]\left[\mathcal{I}_{s^{+}}^{\alpha} \mathcal{D}(s+\varphi(t, s))+\mathcal{I}_{s+\varphi(t, s)^{-}}^{\alpha} \mathcal{D}(s)\right] } \\
\leq & \frac{\Gamma(\alpha)}{\leq(\varphi(t, s))^{\alpha}}\left[\mathcal{I}_{s^{+}}^{\alpha} \mathfrak{Q}_{*} \mathcal{D}(s+\varphi(t, s))+\mathcal{I}_{s+\varphi(t, s)^{-}}^{\alpha} \mathfrak{Q}_{*} \mathcal{D}(s), \mathcal{I}_{s^{+}}^{\alpha} \mathfrak{Q}^{*} \mathcal{D}(s+\varphi(t, s))+\mathcal{I}_{s+\varphi(t, s)^{-}}^{\alpha} \mathfrak{Q}^{*} \mathcal{D}(s)\right],
\end{aligned}
$$

that is

$$
\begin{aligned}
& \frac{\Gamma(\alpha)}{(\varphi(t, s))^{\alpha}} \mathfrak{Q}\left(\frac{2 s+\varphi(t, s)}{2}\right)\left[\mathcal{I}_{s^{+}}^{\alpha} \mathcal{D}(s+\varphi(t, s))+\mathcal{I}_{s+\varphi(t, s)^{-}}^{\alpha} \mathcal{D}(s)\right] \\
& \quad \leq_{p} \frac{\Gamma(\alpha)}{(\varphi(t, s))^{\alpha}}\left[\mathcal{I}_{s^{+}}^{\alpha} \mathfrak{Q D}(s+\varphi(t, s))+\mathcal{I}_{s+\varphi(t, s)^{-}}^{\alpha} \mathfrak{Q D}(s)\right]
\end{aligned}
$$

This completes the proof.

Example 2. We consider the $I-V-F \mathfrak{Q}:[0,2] \rightarrow \mathbb{R}_{I}^{+}$defined by $\mathfrak{Q}(\omega)=[(2-\sqrt{\omega}), 2(2-\sqrt{\omega})]$. Since end point functions $\mathfrak{Q}_{*}(\omega), \mathfrak{Q}^{*}(\omega)$ are preinvex functions with respect to $\varphi(t, s)=t-s$, then $\mathfrak{Q}(\omega)$ is LR-preinvex I-V-F. If

$$
\mathcal{D}(\omega)=\left\{\begin{array}{cc}
\sqrt{\omega}, & \sigma \in[0,1] \\
\sqrt{2-\omega}, & \sigma \in(1,2]
\end{array}\right.
$$

then $\mathcal{D}(2-\omega)=\mathcal{D}(\omega) \geq 0$, for all $\omega \in[0,2]$. Since $\mathfrak{Q}_{*}(\omega)=2-\sqrt{\omega}$ and $\mathfrak{Q}^{*}(\omega)=2(2-\sqrt{\omega})$. If $\alpha=\frac{1}{2}$, then we compute the following:

$$
\begin{gathered}
{\left[\mathcal{I}_{s^{+}}^{\alpha} \mathfrak{Q} \mathcal{D}(s+\varphi(t, s)) \widetilde{+}_{\mathcal{I}_{s+\varphi(t, s)^{-}}^{\alpha}} \mathfrak{Q} \mathcal{D}(s)\right] \leq \frac{\mathfrak{Q}(s)+\mathfrak{Q}(s+\varphi(t, s))}{2}\left[\begin{array}{c}
\mathcal{I}_{s^{+}}^{\alpha} D(s+\varphi(t, s)) \\
+\mathcal{I}_{s+\varphi(t, s)^{\alpha}}^{\alpha} D(s)
\end{array}\right]} \\
\quad \leq_{p} \frac{\mathfrak{Q}(s)+\mathfrak{Q}(t)}{2}\left[\mathcal{I}_{s^{+}}^{\alpha} \mathcal{D}(s+\varphi(t, s))+\mathcal{I}_{s+\varphi(t, s)^{-}}^{\alpha} \mathcal{D}(s)\right] \\
\frac{\mathfrak{Q}_{*}(s)+\mathfrak{Q}_{*}(s+\varphi(t, s))}{2}\left[\mathcal{I}_{s^{+}}^{\alpha} \mathcal{D}(s+\varphi(t, s))+\mathcal{I}_{s+\varphi(t, s)^{-}}^{\alpha} \mathcal{D}(s)\right]=\frac{\pi}{\sqrt{2}}\left(\frac{4-\sqrt{2}}{2}\right) \\
\frac{\mathfrak{Q}^{*}(s) \widetilde{+} \mathfrak{Q}^{*}(s+\varphi(t, s))}{2}\left[\mathcal{I}_{s^{+}}^{\alpha} \mathcal{D}(s+\varphi(t, s))+\mathcal{I}_{s+\varphi(t, s)^{-}}^{\alpha} \mathcal{D}(s)\right]=\frac{\pi}{\sqrt{2}}(4-\sqrt{2}), \\
\quad \frac{\mathfrak{Q}_{*}(s)+\mathfrak{Q}_{*}(t)}{2}\left[\mathcal{I}_{s^{+}}^{\alpha} \mathcal{D}(s+\varphi(t, s))+\mathcal{I}_{s+\varphi(t, s)^{-}}^{\alpha} \mathcal{D}(s)\right]=\frac{\pi}{\sqrt{2}}\left(\frac{4-\sqrt{2}}{2}\right) \\
\frac{\mathfrak{Q}^{*}(s) \widetilde{+} \mathfrak{Q}^{*}(t)}{2}\left[\mathcal{I}_{s^{+}}^{\alpha} \mathcal{D}(s+\varphi(t, s))+\mathcal{I}_{s+\varphi(t, s)^{-}} \mathcal{D}(s)\right]=\frac{\pi}{\sqrt{2}}(4-\sqrt{2}), \\
{\left[\mathcal{I}_{s^{+}}^{\alpha} \mathfrak{Q} * \mathcal{D}(s+\varphi(t, s))+\mathcal{I}_{s+\varphi(t, s)^{-}}^{\alpha} \mathfrak{Q}_{*} \mathcal{D}(s)\right]=\frac{1}{\sqrt{\pi}}\left(2 \pi+\frac{4-8 \sqrt{2}}{3}\right),} \\
{\left[\mathcal{I}_{s^{+}}^{\alpha} \mathfrak{Q}^{*} \mathcal{D}(s+\varphi(t, s))+\mathcal{I}_{s+\varphi(t, s)^{-}}^{\alpha} \mathfrak{Q}^{*} \mathcal{D}(s)\right]=\frac{2}{\sqrt{\pi}}\left(2 \pi+\frac{4-8 \sqrt{2}}{3}\right) .}
\end{gathered}
$$

From (19), (20), and (21), we have

$$
\frac{1}{\sqrt{\pi}}\left[\left(2 \pi+\frac{4-8 \sqrt{2}}{3}\right), 2\left(2 \pi+\frac{4-8 \sqrt{2}}{3}\right)\right] \leq p \frac{\pi}{\sqrt{2}}\left[\frac{4-\sqrt{2}}{2}, 4-\sqrt{2}\right]=\frac{\pi}{\sqrt{2}}\left[\frac{4-\sqrt{2}}{2}, 4-\sqrt{2}\right]
$$

Hence, Theorem 4 is verified.

For Theorem 5, we have

$$
\begin{aligned}
& \mathfrak{Q}_{*}\left(\frac{2 s+\varphi(t, s)}{2}\right)\left[\mathcal{I}_{s^{+}}^{\alpha} \mathcal{D}(s+\varphi(t, s))+\mathcal{I}_{s+\varphi(t, s)^{-}}^{\alpha} \mathcal{D}(s)\right]=\sqrt{\pi}, \\
& \mathfrak{Q}^{*}\left(\frac{2 s+\varphi(t, s)}{2}\right)\left[\mathcal{I}_{s^{+}}^{\alpha} \mathcal{D}(s+\varphi(t, s))+\mathcal{I}_{s+\varphi(t, s)^{-}}^{\alpha} \mathcal{D}(s)\right]=2 \sqrt{\pi} .
\end{aligned}
$$

From (21) and (22), we have

$$
\sqrt{\pi}[1,2] \leq p \frac{1}{\sqrt{\pi}}\left[2 \pi+\frac{4-8 \sqrt{2}}{3}, 2\left(2 \pi+\frac{4-8 \sqrt{2}}{3}\right)\right] .
$$


Remark 3. If $\mathcal{D}(\omega)=1$. Then from Theorem 4 and Theorem 5, we obtain Theorem 3.

Let $\alpha=1$. Then we obtain following new Hermite-Hadamard Fejér type inequality for $L R$-preinvex $I-V-F$,

$$
\mathfrak{Q}\left(\frac{2 s+\varphi(t, s)}{2}\right) \leq_{p} \frac{1}{\int_{\mathcal{S}}^{s+\varphi(t, s)} \mathcal{D}(\omega) d \omega}(I R) \int_{s}^{s+\varphi(t, s)} \mathfrak{Q}(\omega) \mathcal{D}(\omega) d \omega \leq_{p} \frac{\mathfrak{Q}(s)+\mathfrak{Q}(t)}{2}
$$

If $\mathfrak{Q}_{*}(\omega)=\mathfrak{Q}^{*}(\omega)$ with $\varphi(\omega, \mathcal{Z})=\omega-\mathcal{Z}$ and $\mathcal{D}(\omega)=\alpha=1$. Then from Theorem 4 and Theorem 5, we obtain the classical Hermite-Hadamard inequality.

If $\mathfrak{Q}_{*}(\omega)=\mathfrak{Q}^{*}(\omega)$ with $\varphi(\omega, \mathcal{Z})=\omega-\mathcal{Z}$ and $\alpha=1$, then from Theorem 4 and Theorem 5, we obtain the classical Hermite-Hadamard Fejér inequality.

From Theorems 6 and 7, now we obtain several interval fractional integral inequalities linked to interval fractional Hermite-Hadamard type inequality for the product of LRpreinvex $I-V$-Fs.

Theorem 6. Let $\mathfrak{Q}, \mathfrak{S}:[s, s+\varphi(t, s)] \rightarrow \mathbb{R}_{I}^{+}$be two LR-preinvex $I-V$-Fs on $[s, s+\varphi(t, s)]$, such that $\mathfrak{Q}(\omega)=\left[\mathfrak{Q}_{*}(\omega), \mathfrak{Q}^{*}(\omega)\right]$ and $\mathfrak{S}(\omega)=\left[\mathfrak{S}_{*}(\omega), \mathfrak{S}^{*}(\omega)\right]$ for all $\omega \in[s, s+\varphi(t, s)]$. If $\mathfrak{Q} \times \mathfrak{S} \in L\left([s, s+\varphi(t, s)], \mathbb{R}_{I}^{+}\right)$and $\varphi$ satisfies Condition $C$, then

$$
\begin{aligned}
& \frac{\Gamma(\alpha)}{2(\varphi(t, s))^{\alpha}}\left[\mathcal{I}_{s^{+}}^{\alpha} \mathfrak{Q}(s+\varphi(t, s)) \times \mathfrak{S}(s+\varphi(t, s))+\mathcal{I}_{s+\varphi(t, s)^{-}}^{\alpha}(s) \times \mathfrak{S}(s)\right] \\
& \leq_{p}\left(\frac{1}{2}-\frac{\alpha}{(\alpha+1)(\alpha+2)}\right) \Delta(s, s+\varphi(t, s))+\left(\frac{\alpha}{(\alpha+1)(\alpha+2)}\right) \nabla(s, s+\varphi(t, s)) .
\end{aligned}
$$

where $\Delta(s, s+\varphi(t, s))=\mathfrak{Q}(s) \times \mathfrak{S}(s)+\mathfrak{Q}(s+\varphi(t, s)) \times \mathfrak{S}(s+\varphi(t, s)), \nabla(s, s+\varphi(t, s))=$ $\mathfrak{Q}(s) \times \mathfrak{S}(s+\varphi(t, s))+\mathfrak{Q}(s+\varphi(t, s)) \times \mathfrak{S}(s)$, and $\Delta(s, s+\varphi(t, s))=$ $\left[\Delta_{*}(s, s+\varphi(t, s)), \Delta^{*}(s, s+\varphi(t, s))\right]$ and $\nabla(s, t)=\left[\nabla_{*}(s, s+\varphi(t, s)), \nabla^{*}(s, s+\varphi(t, s))\right]$.

Proof. Since $\mathfrak{Q}, \mathfrak{S}$ both are LR-preinvex $I-V-F s$ then, we have

$$
\begin{aligned}
\mathfrak{Q}_{*}(s+(1-\sigma) \varphi(t, s)) & =\mathfrak{Q}_{*}(s+\varphi(t, s)+\sigma \varphi(s, s+\varphi(t, s)) \\
& \leq \sigma \mathfrak{Q}_{*}(s)+(1-\sigma) \mathfrak{Q}_{*}(s+\varphi(t, s)) \\
\mathfrak{Q}^{*}(s+(1-\sigma) \varphi(t, s)) & =\mathfrak{Q}^{*}(s+\varphi(t, s)+\sigma \varphi(s, s+\varphi(t, s)) \\
& \leq \sigma \mathfrak{Q}^{*}(s)+(1-\sigma) \mathfrak{Q}^{*}(s+\varphi(t, s)) .
\end{aligned}
$$

and

$$
\begin{aligned}
\mathfrak{S}_{*}(s+(1-\sigma) \varphi(t, s))= & \mathfrak{S}_{*}(s+\varphi(t, s)+\sigma \varphi(s, s+\varphi(t, s))) \\
& \leq \sigma \mathfrak{S}_{*}(s)+(1-\sigma) \mathfrak{S}_{*}(s+\varphi(t, s)) \\
\mathfrak{S}^{*}(s+(1-\sigma) \varphi(t, s)) & =\mathfrak{S}^{*}(s+\varphi(t, s)+\sigma \varphi(s, s+\varphi(t, s))) \\
& \leq \sigma \mathfrak{S}^{*}(s)+(1-\sigma) \mathfrak{S}^{*}(s+\varphi(t, s)) .
\end{aligned}
$$

From the definition of LR-preinvex $I-V$-Fs it follows that $0 \leq_{p} \mathfrak{Q}(\omega)$ and $0 \leq_{p} \mathfrak{S}(\omega)$, so

$$
\begin{aligned}
\mathfrak{Q}_{*}(s+(1- & \sigma) \varphi(t, s)) \times \mathfrak{S}_{*}(s+(1-\sigma) \varphi(t, s)) \\
\leq & \left(\sigma \mathfrak{Q}_{*}(s)+(1-\sigma) \mathfrak{Q}_{*}(s+\varphi(t, s))\right)\left(\sigma \mathfrak{S}_{*}(s)+(1-\sigma) \mathfrak{S}_{*}(s+\varphi(t, s))\right) \\
& =\sigma^{2} \mathfrak{Q}_{*}(s) \times \mathfrak{S}_{*}(s)+(1-\sigma)^{2} \mathfrak{Q}_{*}(s+\varphi(t, s)) \times \mathfrak{S}_{*}(s+\varphi(t, s)) \\
& +\sigma(1-\sigma) \mathfrak{Q}_{*}(s) \times \mathfrak{S}_{*}(s+\varphi(t, s))+\sigma(1-\sigma) \mathfrak{Q}_{*}(s+\varphi(t, s)) \times \mathfrak{S}_{*}(s) \\
\mathfrak{Q}^{*}(s+(1- & \sigma) \varphi(t, s)) \times \mathfrak{S}^{*}(s+(1-\sigma) \varphi(t, s)) \\
& \leq\left(\sigma \mathfrak{Q}^{*}(s)+(1-\sigma) \mathfrak{Q}^{*}(s+\varphi(t, s))\right)\left(\sigma \mathfrak{S}^{*}(s)+(1-\sigma) \mathfrak{S}^{*}(s+\varphi(t, s))\right) \\
& =\sigma^{2} \mathfrak{Q}^{*}(s) \times \mathfrak{S}^{*}(s)+(1-\sigma)^{2} \mathfrak{Q}^{*}(s+\varphi(t, s)) \times \mathfrak{S}^{*}(s+\varphi(t, s)) \\
& +\sigma(1-\sigma) \mathfrak{Q}^{*}(s) \times \mathfrak{S}^{*}(s+\varphi(t, s))+\sigma(1-\sigma) \mathfrak{Q}^{*}(s+\varphi(t, s)) \times \mathfrak{S}^{*}(s),
\end{aligned}
$$




\section{Analogously, we have}

$$
\begin{aligned}
\mathfrak{Q}_{*}(s+ & \sigma \varphi(t, s)) \times \mathfrak{S}_{*}(s+\sigma \varphi(t, s)) \\
& \leq(1-\sigma)^{2} \mathfrak{Q}_{*}(s) \times \mathfrak{S}_{*}(s)+\sigma^{2} \mathfrak{Q}_{*}(s+\varphi(t, s)) \times \mathfrak{S}_{*}(s+\varphi(t, s)) \\
& +\sigma(1-\sigma) \mathfrak{Q}_{*}(s) \times \mathfrak{S}_{*}(s+\varphi(t, s))+\sigma(1-\sigma) \mathfrak{Q}_{*}(s+\varphi(t, s)) \times \mathfrak{S}_{*}(s) \\
\mathfrak{Q}^{*}(s+ & \sigma \varphi(t, s)) \times \mathfrak{S}^{*}(s+\sigma \varphi(t, s)) \\
& \leq(1-\sigma)^{2} \mathfrak{Q}^{*}(s) \times \mathfrak{S}^{*}(s)+\sigma^{2} \mathfrak{Q}^{*}(s+\varphi(t, s)) \times \mathfrak{S}^{*}(s+\varphi(t, s)) \\
& +\sigma(1-\sigma) \mathfrak{Q}^{*}(s) \times \mathfrak{S}^{*}(s+\varphi(t, s))+\sigma(1-\sigma) \mathfrak{Q}^{*}(s+\varphi(t, s)) \times \mathfrak{S}^{*}(s) .
\end{aligned}
$$

Adding (23) and (24), we have

$$
\begin{aligned}
\mathfrak{Q}_{*}(s+ & (1-\sigma) \varphi(t, s)) \times \mathfrak{S}_{*}(s+(1-\sigma) \varphi(t, s)) \\
& +\mathfrak{Q}_{*}(s+\sigma \varphi(t, s)) \times \mathfrak{S}_{*}(s+\sigma \varphi(t, s)) \\
\leq & {\left[\sigma^{2}+(1-\sigma)^{2}\right]\left[\mathfrak{Q}_{*}(s) \times \mathfrak{S}_{*}(s)+\mathfrak{Q}_{*}(s+\varphi(t, s)) \times \mathfrak{S}_{*}(s+\varphi(t, s))\right] } \\
& +2 \sigma(1-\sigma)\left[\mathfrak{Q}_{*}(s+\varphi(t, s)) \times \mathfrak{S}_{*}(s)+\mathfrak{Q}_{*}(s) \times \mathfrak{S}_{*}(s+\varphi(t, s))\right] \\
\mathfrak{Q}^{*}(s+ & (1-\sigma) \varphi(t, s)) \times \mathfrak{S}^{*}(s+(1-\sigma) \varphi(t, s)) \\
& +\mathfrak{Q}^{*}(s+\sigma \varphi(t, s)) \times \mathfrak{S}^{*}(s+\sigma \varphi(t, s)) \\
\leq & {\left[\sigma^{2}+(1-\sigma)^{2}\right]\left[\mathfrak{Q}^{*}(s) \times \mathfrak{S}^{*}(s)+\mathfrak{Q}^{*}(s+\varphi(t, s)) \times \mathfrak{S}^{*}(s+\varphi(t, s))\right] } \\
& +2 \sigma(1-\sigma)\left[\mathfrak{Q}^{*}(s+\varphi(t, s)) \times \mathfrak{S}^{*}(s)+\mathfrak{Q}^{*}(s) \times \mathfrak{S}^{*}(s+\varphi(t, s))\right] .
\end{aligned}
$$

Taking multiplication of (25) by $\sigma^{\alpha-1}$ and integrating the obtained result with respect to $\sigma$ over $(0,1)$, we have

$$
\begin{aligned}
& \int_{0}^{1} \sigma^{\alpha-1} \mathfrak{Q}_{*}(s+(1-\sigma) \varphi(t, s)) \times \mathfrak{S}_{*}(s+(1-\sigma) \varphi(t, s)) \\
&+ \sigma^{\alpha-1} \mathfrak{Q}_{*}(s+\sigma \varphi(t, s)) \times \mathfrak{S}_{*}(s+\sigma \varphi(t, s)) d \sigma \\
& \leq \Delta_{*}(s, s+\varphi(t, s)) \int_{0}^{1} \sigma^{\alpha-1}\left[\sigma^{2}+(1-\sigma)^{2}\right] d \sigma+2 \nabla_{*}(s, s+\varphi(t, s)) \int_{0}^{1} \sigma^{\alpha-1} \sigma(1-\sigma) d \sigma \\
& \int_{0}^{1} \sigma^{\alpha-1} \mathfrak{Q}^{*}(s+(1-\sigma) \varphi(t, s)) \times \mathfrak{S}^{*}(s+(1-\sigma) \varphi(t, s)) \\
&+ \sigma^{\alpha-1} \mathfrak{Q}^{*}(s+\sigma \varphi(t, s)) \times \mathfrak{S}^{*}(s+\sigma \varphi(t, s)) d \sigma \\
&\left.\leq \Delta^{*}(s, s+\varphi(t, s))\right) \int_{0}^{1} \sigma^{\alpha-1}\left[\sigma^{2}+(1-\sigma)^{2}\right] d \sigma+2 \nabla^{*}(s, s+\varphi(t, s)) \int_{0}^{1} \sigma^{\alpha-1} \sigma(1-\sigma) d \sigma .
\end{aligned}
$$

It follows that,

$$
\begin{aligned}
& \frac{\Gamma(\alpha)}{(\varphi(t, s))^{\alpha}}\left[\mathcal{I}_{s^{+}}^{\alpha} \mathfrak{Q}_{*}(s+\varphi(t, s)) \times \mathfrak{S}_{*}(s+\varphi(t, s))+\mathcal{I}_{s+\varphi(t, s)^{-}}^{\alpha} \mathfrak{Q}_{*}(s) \times \mathfrak{S}_{*}(s)\right] \\
& \quad \leq \frac{2}{\alpha}\left(\frac{1}{2}-\frac{\alpha}{(\alpha+1)(\alpha+2)}\right) \Delta_{*}(s, s+\varphi(t, s))+\frac{2}{\alpha}\left(\frac{\alpha}{(\alpha+1)(\alpha+2)}\right) \nabla_{*}(s, s+\varphi(t, s)) \\
& \frac{\Gamma(\alpha)}{(\varphi(t, s))^{\alpha}}\left[\mathcal{I}_{s^{+}}^{\alpha} \mathfrak{Q}^{*}(s+\varphi(t, s)) \times \mathfrak{S}^{*}(s+\varphi(t, s))+\mathcal{I}_{s+\varphi(t, s)^{-}}^{\alpha} \mathfrak{Q}^{*}(s) \times \mathfrak{S}^{*}(s)\right] \\
& \quad \leq \frac{2}{\alpha}\left(\frac{1}{2}-\frac{\alpha}{(\alpha+1)(\alpha+2)}\right) \Delta^{*}(s, s+\varphi(t, s))+\frac{2}{\alpha}\left(\frac{\alpha}{(\alpha+1)(\alpha+2)}\right) \nabla^{*}(s, s+\varphi(t, s))
\end{aligned}
$$

that is

$$
\begin{aligned}
\frac{\Gamma(\alpha)}{(\varphi(t, s))^{\alpha}}\left[\mathcal{I}_{s^{+}}^{\alpha} \mathfrak{Q}_{*}(s+\varphi(t, s)) \times \mathfrak{S}_{*}(s+\varphi(t, s))\right. & \left.+\mathcal{I}_{s+\varphi(t, s)}^{\alpha} \mathfrak{Q}_{*}(s) \times \mathfrak{S}_{*}(s), \mathcal{I}_{s^{+}}^{\alpha} \mathfrak{Q}^{*}(s+\varphi(t, s)) \times \mathfrak{S}^{*}(s+\varphi(t, s))+\mathcal{I}_{s+\varphi(t, s)^{-}}^{\alpha} \mathfrak{Q}^{*}(s) \times \mathfrak{S}^{*}(s)\right] \\
\leq_{p} & \frac{2}{\alpha}\left(\frac{1}{2}-\frac{\alpha}{(\alpha+1)(\alpha+2)}\right)\left[\Delta_{*}(s, s+\varphi(t, s)), \Delta^{*}(s, s+\varphi(t, s))\right] \\
& +\frac{2}{\alpha}\left(\frac{\alpha}{(\alpha+1)(\alpha+2)}\right)\left[\nabla_{*}(s, s+\varphi(t, s)), \nabla^{*}(s, s+\varphi(t, s))\right] .
\end{aligned}
$$


Thus,

$$
\begin{gathered}
\frac{\Gamma(\alpha)}{2(\varphi(t, s))^{\alpha}}\left[\mathcal{I}_{s^{+}}^{\alpha} \mathfrak{Q}(s+\varphi(t, s)) \times \mathfrak{S}(s+\varphi(t, s))+\mathcal{I}_{s+\varphi(t, s)^{-}}^{\alpha} \mathfrak{Q}(s) \times \mathfrak{S}(s)\right] \\
\leq_{p}\left(\frac{1}{2}-\frac{\alpha}{(\alpha+1)(\alpha+2)}\right) \Delta(s, s+ \\
\varphi(t, s))+\left(\frac{\alpha}{(\alpha+1)(\alpha+2)}\right) \nabla(s, s+\varphi(t, s)) .
\end{gathered}
$$

and the theorem has been established.

Example 3. Let $[s, s+\varphi(t, s)]=[0, \varphi(2,0)], \alpha=\frac{1}{2}, \mathfrak{Q}(\omega)=\left[\frac{\omega}{2}, \frac{3 \omega}{2}\right]$, and $\mathfrak{S}(\omega)=[\omega, 3 \omega]$. Since left and right end point functions $\mathfrak{Q}_{*}(\omega)=\frac{\omega}{2}, \mathfrak{Q}^{*}(\omega)=\frac{3 \omega}{2}, \mathfrak{S}_{*}(\omega)=\omega$, and $\mathfrak{S}^{*}(\omega)=3 \omega$ are $L R$-preinvex functions with respect to $\varphi(t, s)=t-s$, then $\mathfrak{Q}(\omega)$ and $\mathfrak{S}(\omega)$ both are $L R$ preinvex $I-V-F$. We clearly see that $\mathfrak{Q}(\omega) \times \mathfrak{S}(\omega) \in L\left([s, s+\varphi(t, s)], \mathbb{R}_{I}^{+}\right)$and

$$
\begin{gathered}
\frac{\Gamma(1+\alpha)}{2(\varphi(t, s))^{\alpha}}\left[\mathcal{I}_{s^{+}}^{\alpha} \mathfrak{Q}_{*}(s+\varphi(t, s)) \times \mathfrak{S}_{*}(s+\varphi(t, s))+\mathcal{I}_{s+\varphi(t, s)^{-}}^{\alpha} \mathfrak{Q}_{*}(s) \times \mathfrak{S}_{*}(s)\right] \\
=\frac{\Gamma\left(\frac{3}{2}\right)}{2 \sqrt{2}} \frac{1}{\sqrt{\pi}} \int_{0}^{\varphi(2,0)}(2-\omega)^{\frac{-1}{2}}\left(\frac{1}{2} \cdot \omega^{2}\right) d \omega+\frac{\Gamma\left(\frac{3}{2}\right)}{2 \sqrt{2}} \frac{1}{\sqrt{\pi}} \int_{0}^{\varphi(2,0)}(\omega)^{\frac{-1}{2}}\left(\frac{1}{2} \cdot \omega^{2}\right) d \omega \approx 0.7333, \\
\frac{\Gamma(1+\alpha)}{2(\varphi(t, s))^{\alpha}}\left[\mathcal{I}_{s^{+}}^{\alpha} \mathfrak{Q}^{*}(s+\varphi(t, s)) \times \mathfrak{S}^{*}(s+\varphi(t, s))+\mathcal{I}_{s+\varphi(t, s)^{-}}^{\alpha} \mathfrak{Q}^{*}(s) \times \mathfrak{S}^{*}(s)\right] \\
=\frac{\Gamma\left(\frac{3}{2}\right)}{2 \sqrt{2}} \frac{1}{\sqrt{\pi}} \int_{0}^{\varphi(2,0)}(2-\omega)^{\frac{-1}{2}} \cdot \frac{9}{2} \omega^{2} d \omega+\frac{\Gamma\left(\frac{3}{2}\right)}{2 \sqrt{2}} \frac{1}{\sqrt{\pi}} \int_{0}^{\varphi(2,0)}(\omega)^{\frac{-1}{2}} \cdot \frac{9}{2} \omega^{2} d \omega \approx 6.5997,
\end{gathered}
$$

Note that

$$
\begin{gathered}
\left(\frac{1}{2}-\frac{\alpha}{(\alpha+1)(\alpha+2)}\right) \Delta_{*}(s, s+\varphi(t, s))=\left[\mathfrak{Q}_{*}(s) \times \mathfrak{S}_{*}(s)+\mathfrak{Q}_{*}(s+\varphi(t, s)) \times \mathfrak{S}_{*}(s+\varphi(t, s))\right] \\
=\frac{11}{15} \\
\left(\frac{1}{2}-\frac{\alpha}{(\alpha+1)(\alpha+2)}\right) \Delta^{*}(s, s+\varphi(t, s))=\left[\mathfrak{Q}^{*}(s) \times \mathfrak{S}^{*}(s)+\mathfrak{Q}^{*}(s+\varphi(t, s)) \times \mathfrak{S}^{*}(s+\varphi(t, s))\right] \\
=\frac{33}{5}, \\
\left(\frac{\alpha}{(\alpha+1)(\alpha+2)}\right) \nabla_{*}(s, s+\varphi(t, s))=\left[\mathfrak{Q}_{*}(s) \times \mathfrak{S}_{*}(s+\varphi(t, s))+\mathfrak{Q}_{*}(s+\varphi(t, s)) \times \mathfrak{S}_{*}(s)\right] \\
=\frac{2}{15}(0), \\
\left(\frac{\alpha}{(\alpha+1)(\alpha+2)}\right) \nabla_{*}(s, s+\varphi(t, s))=\left[\mathfrak{Q}^{*}(s) \times \mathfrak{S}^{*}(s+\varphi(t, s))+\mathfrak{Q}^{*}(s+\varphi(t, s)) \times \mathfrak{S}^{*}(s)\right] \\
=\frac{2}{15}(0) .
\end{gathered}
$$

Therefore, we have

$$
\left(\frac{1}{2}-\frac{\alpha}{(\alpha+1)(\alpha+2)}\right) \Delta(s, s+\varphi(t, s))+\left(\frac{\alpha}{(\alpha+1)(\alpha+2)}\right) \nabla(s, s+\varphi(t, s))=\left[\frac{11}{15}, \frac{33}{5}\right]+\frac{2}{15}[0,0]=\left[\frac{11}{15}, \frac{33}{5}\right] .
$$

It follows that

$$
[0.7333, \quad 6.5997] \leq_{p}\left[\frac{11}{15}, \frac{33}{5}\right]
$$

and Theorem 6 has been demonstrated.

Theorem 7. Let $\mathfrak{Q}, \mathfrak{S}:[s, s+\varphi(t, s)] \rightarrow \mathbb{R}_{I}^{+}$be two LR-preinvex I-V-Fs, such that $\mathfrak{Q}(\omega)=$ $\left[\mathfrak{Q}_{*}(\omega), \mathfrak{Q}^{*}(\omega)\right]$ and $\mathfrak{S}(\omega)=\left[\mathfrak{S}_{*}(\omega), \mathfrak{S}^{*}(\omega)\right]$ for all $\omega \in[s, s+\varphi(t, s)]$. If $\mathfrak{Q} \times \mathfrak{S} \in$ $L\left([s, s+\varphi(t, s)], \mathbb{R}_{I}^{+}\right)$and $\varphi$ satisfies Condition $C$, then

$$
\begin{aligned}
& \frac{1}{\alpha} \mathfrak{Q}\left(\frac{2 s+\varphi(t, s)}{2}\right) \times \mathfrak{S}\left(\frac{2 s+\varphi(t, s)}{2}\right) \leq_{p} \frac{\Gamma(\alpha+1)}{4(\varphi(t, s))^{\alpha}}\left[\begin{array}{c}
\mathcal{I}_{s^{+}}^{\alpha} \mathfrak{Q}(s+\varphi(t, s)) \times \mathfrak{S}(s+\varphi(t, s)) \\
+\mathcal{I}_{s+\varphi(t, s)^{\alpha}}^{\alpha} \mathfrak{Q}(s) \times \mathfrak{S}(s)
\end{array}\right] \\
& \times \frac{1}{2 \alpha}\left(\frac{1}{2}-\frac{\alpha}{(\alpha+1)(\alpha+2)}\right) \nabla(s, s+\varphi(t, s))+\frac{1}{2 \alpha}\left(\frac{\alpha}{(\alpha+1)(\alpha+2)}\right) \Delta(s, s+\varphi(t, s)) .
\end{aligned}
$$


where $\Delta(s, s+\varphi(t, s))=\mathfrak{Q}(s) \times \mathfrak{S}(s) \times \mathfrak{Q}(s+\varphi(t, s)) \times \mathfrak{S}(s+\varphi(t, s))$, $\nabla(s, t)=\mathfrak{Q}(s) \times \mathfrak{S}(s+\varphi(t, s))+\mathfrak{Q}(s+\varphi(t, s)) \times \mathfrak{S}(s)$, and $\Delta(s, s+\varphi(t, s))=$ $\left[\Delta_{*}(s, s+\varphi(t, s)), \Delta^{*}(s, s+\varphi(t, s))\right], \quad$ and $\quad \nabla(s, s+\varphi(t, s))$ $\left[\nabla_{*}(s, s+\varphi(t, s)), \nabla^{*}(s, s+\varphi(t, s))\right]$.

Proof. Consider $\mathfrak{Q}, \mathfrak{S}:[s, s+\varphi(t, s)] \rightarrow \mathbb{R}_{I}^{+}$are LR-preinvex I-V-Fs. Then by hypothesis, we have

$$
\begin{aligned}
& \mathfrak{Q}_{*}\left(\frac{2 s+\varphi(t, s)}{2}\right) \times \mathfrak{S}_{*}\left(\frac{2 s+\varphi(t, s)}{2}\right) \\
& \mathfrak{Q}^{*}\left(\frac{2 s+\varphi(t, s)}{2}\right) \times \mathfrak{S}^{*}\left(\frac{2 s+\varphi(t, s)}{2}\right) \\
& \leq \frac{1}{4}\left[\begin{array}{c}
\mathfrak{Q}_{*}(s+(1-\sigma) \varphi(t, s)) \times \mathfrak{S}_{*}(s+(1-\sigma) \varphi(t, s)) \\
+\mathfrak{Q}_{*}(s+(1-\sigma) \varphi(t, s)) \times \mathfrak{S}_{*}(s+\sigma \varphi(t, s))
\end{array}\right] \\
& +\frac{1}{4}\left[\begin{array}{c}
\mathfrak{Q}_{*}(s+\sigma \varphi(t, s)) \times \mathfrak{S}_{*}(s+(1-\sigma) \varphi(t, s)) \\
+\mathfrak{Q}_{*}(s+\sigma \varphi(t, s)) \times \mathfrak{S}_{*}(s+\sigma \varphi(t, s))
\end{array}\right] \\
& \leq \frac{1}{4}\left[\begin{array}{c}
\mathfrak{Q}^{*}(s+(1-\sigma) \varphi(t, s)) \times \mathfrak{S}^{*}(s+(1-\sigma) \varphi(t, s)) \\
+\mathfrak{Q}^{*}(s+(1-\sigma) \varphi(t, s)) \times \mathfrak{S}^{*}(s+\sigma \varphi(t, s))
\end{array}\right] \\
& +\frac{1}{4}\left[\begin{array}{c}
\mathfrak{Q}^{*}(s+\sigma \varphi(t, s)) \times \mathfrak{S}^{*}(s+(1-\sigma) \varphi(t, s)) \\
+\mathfrak{Q}^{*}(s+\sigma \varphi(t, s)) \times \mathfrak{S}^{*}(s+\sigma \varphi(t, s))
\end{array}\right], \\
& \leq \frac{1}{4}\left[\begin{array}{c}
\mathfrak{Q}_{*}(s+(1-\sigma) \varphi(t, s)) \times \mathfrak{S}_{*}(s+(1-\sigma) \varphi(t, s)) \\
+\mathfrak{Q}_{*}(s+\sigma \varphi(t, s)) \times \mathfrak{S}_{*}(s+\sigma \varphi(t, s))
\end{array}\right] \\
& +\frac{1}{4}\left[\begin{array}{c}
\left(\sigma \mathfrak{Q}_{*}(s)+(1-\sigma) \mathfrak{Q}_{*}(s+\varphi(t, s))\right) \\
\times\left((1-\sigma) \mathfrak{S}_{*}(s)+\sigma \mathfrak{S}_{*}(s+\varphi(t, s))\right) \\
+\left((1-\sigma) \mathfrak{Q}_{*}(s)+\sigma \mathfrak{Q}_{*}(s+\varphi(t, s))\right) \\
\times\left(\sigma \mathfrak{S}_{*}(s)+(1-\sigma) \mathfrak{S}_{*}(s+\varphi(t, s))\right)
\end{array}\right] \\
& \leq \frac{1}{4}\left[\begin{array}{c}
\mathfrak{Q}^{*}(s+(1-\sigma) \varphi(t, s)) \times \mathfrak{S}^{*}(s+(1-\sigma) \varphi(t, s)) \\
+\mathfrak{Q}^{*}(s+\sigma \varphi(t, s)) \times \mathfrak{S}^{*}(s+\sigma \varphi(t, s))
\end{array}\right] \\
& +\frac{1}{4}\left[\begin{array}{c}
\left(\sigma \mathfrak{Q}^{*}(s)+(1-\sigma) \mathfrak{Q}^{*}(s+\varphi(t, s))\right) \\
\times\left((1-\sigma) \mathfrak{S}^{*}(s)+\sigma \mathfrak{S}^{*}(s+\varphi(t, s))\right) \\
+\left((1-\sigma) \mathfrak{Q}^{*}(s)+\sigma \mathfrak{Q}^{*}(s+\varphi(t, s))\right) \\
\times\left(\sigma \mathfrak{S}^{*}(s)+(1-\sigma) \mathfrak{S}^{*}(s+\varphi(t, s))\right)
\end{array}\right], \\
& =\frac{1}{4}\left[\begin{array}{c}
\mathfrak{Q}_{*}(s+(1-\sigma) \varphi(t, s)) \times \mathfrak{S}_{*}(s+(1-\sigma) \varphi(t, s)) \\
+\mathfrak{Q}_{*}(s+\sigma \varphi(t, s)) \times \mathfrak{S}_{*}(s+\sigma \varphi(t, s))
\end{array}\right] \\
& +\frac{1}{4}\left[\begin{array}{c}
\left\{\sigma^{2}+(1-\sigma)^{2}\right\} \nabla_{*}(s, s+\varphi(t, s)) \\
+\{\sigma(1-\sigma)+(1-\sigma) \sigma\} \Delta_{*}(s, s+\varphi(t, s))
\end{array}\right] \\
& =\frac{1}{4}\left[\begin{array}{c}
\mathfrak{Q}^{*}(s+(1-\sigma) \varphi(t, s)) \times \mathfrak{S}^{*}(s+(1-\sigma) \varphi(t, s)) \\
+\mathfrak{Q}^{*}(s+\sigma \varphi(t, s)) \times \mathfrak{S}^{*}(s+\sigma \varphi(t, s))
\end{array}\right] \\
& +\frac{1}{4}\left[\begin{array}{c}
\left\{\sigma^{2}+(1-\sigma)^{2}\right\} \nabla^{*}(s, s+\varphi(t, s)) \\
+\{\sigma(1-\sigma)+(1-\sigma) \sigma\} \Delta^{*}(s, s+\varphi(t, s))
\end{array}\right] \text {. }
\end{aligned}
$$


Taking multiplication of (26) with $\sigma^{\alpha-1}$ and integrating over $(0,1)$, we obtain

$$
\begin{aligned}
& \frac{1}{\alpha} \mathfrak{Q}_{*}\left(\frac{2 s+\varphi(t, s)}{2}\right) \times \mathfrak{S}_{*}\left(\frac{2 s+\varphi(t, s)}{2}\right) \\
& \leq \frac{1}{4(\varphi(t, s))^{\alpha}}\left[\begin{array}{c}
\int_{s}^{s+\varphi(t, s)}(s+\varphi(t, s)-\omega)^{\alpha-1} \mathfrak{Q}_{*}(\omega) \times \mathfrak{S}_{*}(\omega) d \omega \\
+\int_{s}^{s+\varphi(t, s)}(\mathcal{Z}-s)^{\alpha-1} \mathfrak{Q}_{*}(\mathcal{Z}) \times \mathfrak{S}_{*}(\mathcal{Z}) d \mathcal{Z}
\end{array}\right] \\
& +\frac{1}{2 \alpha}\left(\frac{1}{2}-\frac{\alpha}{(\alpha+1)(\alpha+2)}\right) \nabla_{*}(s, s+\varphi(t, s))+\frac{1}{2 \alpha}\left(\frac{\alpha}{(\alpha+1)(\alpha+2)}\right) \Delta_{*}(s, s+\varphi(t, s)) \\
& =\frac{\Gamma(\alpha+1)}{4(\varphi(t, s))^{\alpha}}\left[\mathcal{I}_{s^{+}}^{\alpha} \mathfrak{Q}_{*}(s+\varphi(t, s)) \times \mathfrak{S}_{*}(s+\varphi(t, s))+\mathcal{I}_{s+\varphi(t, s)^{-}}^{\alpha} \mathfrak{Q}_{*}(s) \times \mathfrak{S}_{*}(s)\right] \\
& +\frac{1}{2 \alpha}\left(\frac{1}{2}-\frac{\alpha}{(\alpha+1)(\alpha+2)}\right) \nabla_{*}(s, s+\varphi(t, s))+\frac{1}{2 \alpha}\left(\frac{\alpha}{(\alpha+1)(\alpha+2)}\right) \Delta_{*}(s, s+\varphi(t, s)) \\
& \frac{1}{\alpha} \mathfrak{Q}^{*}\left(\frac{2 s+\varphi(t, s)}{2}\right) \times \mathfrak{S}^{*}\left(\frac{2 s+\varphi(t, s)}{2}\right) \\
& \leq \frac{1}{4(\varphi(t, s))^{\alpha}}\left[\begin{array}{c}
\int_{\mathcal{s}}^{s+\varphi(t, s)}(s+\varphi(t, s)-\omega)^{\alpha-1} \mathfrak{Q}^{*}(\omega) \times \mathfrak{S}^{*}(\omega) d \omega \\
+\int_{\mathcal{S}}^{s+\varphi(t, s)}(\mathcal{Z}-s)^{\alpha-1} \mathfrak{Q}^{*}(\mathcal{Z}) \times \mathfrak{S}^{*}(\mathcal{Z}) d \mathcal{Z}
\end{array}\right] \\
& +\frac{1}{2 \alpha}\left(\frac{1}{2}-\frac{\alpha}{(\alpha+1)(\alpha+2)}\right) \nabla^{*}(s, s+\varphi(t, s))+\frac{1}{2 \alpha}\left(\frac{\alpha}{(\alpha+1)(\alpha+2)}\right) \Delta^{*}(s, s+\varphi(t, s)) \\
& =\frac{\Gamma(\alpha+1)}{4(\varphi(t, s))^{\alpha}}\left[\mathcal{I}_{s^{+}}^{\alpha} \mathfrak{Q}^{*}(s+\varphi(t, s)) \times \mathfrak{S}^{*}(s+\varphi(t, s))+\mathcal{I}_{s+\varphi(t, s)^{-}}^{\alpha} \mathfrak{Q}^{*}(s) \times \mathfrak{S}^{*}(s)\right] \\
& +\frac{1}{2 \alpha}\left(\frac{1}{2}-\frac{\alpha}{(\alpha+1)(\alpha+2)}\right) \nabla^{*}(s, s+\varphi(t, s))+\frac{1}{2 \alpha}\left(\frac{\alpha}{(\alpha+1)(\alpha+2)}\right) \Delta^{*}(s, s+\varphi(t, s)) \text {, }
\end{aligned}
$$

that is

$$
\begin{aligned}
& \frac{1}{\alpha} \mathfrak{Q}\left(\frac{2 s+\varphi(t, s)}{2}\right) \times \mathfrak{S}\left(\frac{2 s+\varphi(t, s)}{2}\right) \leq_{p} \frac{\Gamma(\alpha+1)}{4(\varphi(t, s))^{\alpha}}\left[\begin{array}{c}
\mathcal{I}_{s^{+}}^{\alpha} \mathfrak{Q}(s+\varphi(t, s)) \times \mathfrak{S}(s+\varphi(t, s)) \\
+\mathcal{I}_{s+\varphi(t, s)^{\alpha}}^{\alpha} \mathfrak{Q}(s) \times \mathfrak{S}(s)
\end{array}\right]+\frac{1}{2 \alpha}\left(\frac{1}{2}-\frac{\alpha}{(\alpha+1)(\alpha+2)}\right) \\
& \nabla(s, s+\varphi(t, s))+\frac{1}{2 \alpha}\left(\frac{\alpha}{(\alpha+1)(\alpha+2)}\right) \Delta(s, s+\varphi(t, s)) .
\end{aligned}
$$

Hence, the required result.

\section{Conclusions and Future Plan}

The LR-preinvex $I-V$-Fs were explored in this paper, a novel family of preinvex functions. Following that, we identified a connection between Riemann-Liouville fractional integral inequalities and LR-preinvex I- $V$-Fs. Moreover, we derived several known and new particular examples as applications of LR-preinvex $I-V$-Fs and Riemann-Liouville fractional integral inequalities. In future study, we will examine this notion for generalized LR-preinvex $I-V$-Fs and $F-I-V$-Fs using generalized interval and fuzzy Riemann-Liouville fractional operators.

Author Contributions: Conceptualization, M.B.K.; validation, M.A.N. and T.A.; formal analysis, M.A.N. and T.A.; investigation, M.B.K. and T.A.; resources, M.B.K., S.M.A., and B.A.; writingoriginal draft, M.B.K. and M.A.N.; writing-review and editing, M.B.K. and T.A.; visualization, M.B.K., A.A.A.M. and T.A.; supervision, M.B.K. and T.A.; project administration, M.A.N., A.A.A.M., S.M.A. and T.A. All authors have read and agreed to the published version of the manuscript.

Funding: Not Applicable.

Data Availability Statement: Not Applicable.

Acknowledgments: The authors would like to thank the Rector, COMSATS University Islamabad, Islamabad, Pakistan, for providing excellent research and academic environments. This Research was supported by Taif University Researchers Supporting Project Number (TURSP-2020/48), Taif University, Taif, Saudi Arabia and the authors T. Abdeljawad and B. Abdalla would like to thank Prince Sultan University for paying the APC and for the support through the TAS research lab.

Conflicts of Interest: The authors declare no conflict of interest. 


\section{References}

1. Cheung, W.-S. Some generalized Opial-type inequalities. J. Math. Anal. Appl. 1991, 162, 317-321. [CrossRef]

2. Farid, G.; Rehman, A.U.; Bibi, S.; Chu, Y.M. Refinements of two fractional versions of Hadamard inequalities for Caputo fractional derivatives and related results. Open J. Math. Sci. 2021, 5, 1-10. [CrossRef]

3. Khan, M.A.; Begum, S.; Khurshid, Y.; Chu, Y.M. Ostrowski type inequalities involving conformable fractional integrals. J. Inequal. Appl. 2018, 2018, 70. [CrossRef] [PubMed]

4. Sarikaya, M.Z.; Bilişik, C.C.; Tunç, T. On Hardy type inequalities via k-fractional integrals. TWMS J. Appl. Eng. Math. 2020, 10, 443-451.

5. Dahmani, Z.; Tabharit, L.; Taf, S. New generalizations of Grüss inequality using Riemann-Liouville fractional integrals. Bull. Math. Anal. Appl. 2010, 2, 93-99.

6. Set, E.; Akdemir, A.O.; Ozata, F. Grüss type inequalities for fractional integral operator involving the extended generalized Mittag-Leffler function. Appl. Comput. Math. 2020, 19, 402-414.

7. Ișcan, I. Hermite-Hadamard type inequalities for harmonically convex functions. Hacet. J. Math. Stat. 2014, 43, 935-942. [CrossRef]

8. Chen, F.; Wu, S. Fejér and Hermite-Hadamard type inqequalities for harmonically convex functions. J. App.l Math. 2014, 2014, 386806.

9. Chen, F. Extensions of the Hermite-Hadamard inequality for harmonically convex functions via fractional integrals. Appl. Math. Comput. 2015, 268, 121-128. [CrossRef]

10. Allahviranloo, T.; Salahshour, S.; Abbasbandy, S. Explicit solutions of fractional differential equations with uncertainty. Soft Comput. Fus. Found. Meth. Appl. 2012, 16, 297-302. [CrossRef]

11. Costa, T.M. Jensen's inequality type integral for fuzzy-interval-valued functions. Fuzzy Sets Syst. 2017, 327, 31-47. [CrossRef]

12. Costa, T.M.; Roman-Flores, H. Some integral inequalities for fuzzy-interval-valued functions. Inform. Sci. 2017, 420, 110-125. [CrossRef]

13. Román-Flores, H.; Chalco-Cano, Y.; Lodwick, W.A. Some integral inequalities for interval-valued functions. Comput. Appl. Math. 2018, 37, 1306-1318. [CrossRef]

14. Roman-Flores, H.; Chalco-Cano, Y.; Silva, G.N. A Note on Gronwall Type Inequality for Interval-Valued Functions. In Proceedings of the 2013 Joint IFSA World Congress and NAFIPS Annual Meeting (IFSA/NAFIPS), Edmonton, AB, Canada, 24-28 June 2013; Volume 35, pp. 1455-1458.

15. Chalco-Cano, Y.; Flores-Franulič, A.; Román-Flores, H. Ostrowski type inequalities for interval-valued functions using generalized Hukuhara derivative. Comput. Appl. Math. 2012, 31, 457-472.

16. Chalco-Cano, Y.; Lodwick, W.A.; Condori-Equice, W. Ostrowski type inequalities and applications in numerical integration for interval-valued functions. Soft Comput. 2015, 19, 3293-3300. [CrossRef]

17. Nikodem, K.; Snchez, J.L.; Snchez, L. Jensen and Hermite-Hadamard inequalities for strongly convex set-valued maps. Math. Aeterna 2014, 4, 979-987.

18. Matkowski, J.; Nikodem, K. An integral Jensen inequality for convex multifunctions. Results Math. 1994, 26, 348-353. [CrossRef]

19. Zhao, D.; An, T.; Ye, G.; Liu, W. Chebyshev type inequalities for interval-valued functions. Fuzzy Sets Syst. 2020, 396, 82-101. [CrossRef]

20. Zhao, D.F.; An, T.Q.; Ye, G.J.; Liu, W. New Jensen and Hermite-Hadamard type inequalities for h-convex interval-valued functions. J. Inequal. Appl. 2018, 2018, 1-14. [CrossRef]

21. Zhang, D.; Guo, C.; Chen, D.; Wang, G. Jensen's inequalities for set-valued and fuzzy set-valued functions. Fuzzy Sets Syst. 2020, 2020, 1-27. [CrossRef]

22. Budak, H.; Tunç, T.; Sarikaya, M. Fractional Hermite-Hadamard-type inequalities for interval-valued functions. Proc. Am. Math. Soc. 2020, 148, 705-718. [CrossRef]

23. Zhao, D.; Ali, M.A.; Murtaza, G.; Zhang, Z. On the Hermite-Hadamard inequalities for interval-valued coordinated convex functions. Adv. Differ. Equ. 2020, 2020, 1-14. [CrossRef]

24. Kara, H.; Ali, M.A.; Budak, H. Hermite-Hadamard-type inequalities for interval-valued coordinated convex functions involving generalized fractional integrals. Math. Methods Appl. Sci. 2021, 44, 104-123. [CrossRef]

25. Shi, F.; Ye, G.; Zhao, D.; Liu, W. Some fractional Hermite-Hadamard-type inequalities for interval-valued coordinated functions. Adv. Differ. Equ. 2021, 2021, 1-17. [CrossRef]

26. Dragomir, S.S. On the Hadamard's inequlality for convex functions on the co-ordinates in a rectangle from the plane. Taizwan J. Math. 2001, 2001, 775-788. [CrossRef]

27. Khan, M.B.; Noor, M.A.; Noor, K.I.; Chu, Y.M. New Hermite-Hadamard type inequalities for (h1, h2)-convex fuzzy-interval-valued functions. Adv. Differ. Equ. 2021, 2021, 6-20. [CrossRef]

28. Khan, M.B.; Noor, M.A.; Abdullah, L.; Chu, Y.M. Some new classes of preinvex fuzzy-interval-valued functions and inequalities. Int. J. Comput. Intell. Syst. 2021, 14, 1403-1418. [CrossRef]

29. Liu, P.; Khan, M.B.; Noor, M.A.; Noor, K.I. New Hermite-Hadamard and Jensen inequalities for log-s-convex fuzzy-interval-valued functions in the second sense. Complex. Intell. Syst. 2021, 2021,1-15. [CrossRef]

30. Sana, G.; Khan, M.B.; Noor, M.A.; Mohammed, P.O.; Chu, Y.M. Harmonically convex fuzzy-interval-valued functions and fuzzy-interval Riemann-Liouville fractional integral inequalities. Int. J. Comput. Intell. Syst. 2021, 14, 1809-1822. [CrossRef]

31. Kulish, U.; Miranker, W. Computer Arithmetic in Theory and Practice; Academic Press: New York, NY, USA, 2014. 
32. Kaleva, O. Fuzzy differential equations. Fuzzy Sets Syst. 1987, 24, 301-317. [CrossRef]

33. Nanda, N.; Kar, K. Convex fuzzy mappings. Fuzzy Sets Syst. 1992, 48, 129-132. [CrossRef]

34. Noor, M.A. Fuzzy preinvex functions. Fuzzy Sets Syst. 1994, 64, 95-104. [CrossRef]

35. Fang, Z.-B.; Shi, R.-J. On the (p, h)-convex function and some integral inequalities. J. Inequal. Appl. 2014, 2014, 45. [CrossRef]

36. Kunt, M.; İşcan, İ. Hermite-Hadamard-Fejér type inequalities for p-convex functions. Arab. J. Math. Sci. 2017, 23, 215-230. [CrossRef]

37. Fejér, L. Über die Fourierreihen II. Math. Naturwise. Anz. Ungar. Akad. Wiss. 1906, 24, 369-390.

38. Srivastava, H.M.; El-Deeb, S.M. Fuzzy differential subordinations based upon the Mittag-Leffler type Borel distribution. Symmetry 2021, 13, 1023. [CrossRef]

39. Noor, M.A.; Noor, K.I.; Awan, M.U.; Costache, S. Some integral inequalities for harmonically h-convex functions. Politehn. Univ. Bucharest Sci. Bull. Ser. A Appl. Math. Phys. 2015, 77, 5-16.

40. Khan, M.B.; Noor, M.A.; Noor, K.I. Some Inequalities for LR- $\left(h_{1}, h_{2}\right)$-Convex Interval-Valued Functions by Means of Pseudo Order Relation. Int. J. Comput. Intell. Syst. 2021, 14, 180.

41. Lupulescu, V. Fractional calculus for interval-valued functions. Fuzzy Sets Syst. 2015, 265, 63-85. [CrossRef]

42. Moore, R.E. Interval Analysis; Prentice Hall: Englewood Cliffs, NJ, USA, 1966.

43. Khan, M.B.; Mohammed, P.O.; Noor, M.A.; Baleanu, D.; Guirao, J. Some New Fractional Estimates of Inequalities for LR-p-Convex Interval-Valued Functions by Means of Pseudo Order Relation. Axioms 2021, 10, 175. [CrossRef]

44. Mohan, S.R.; Neogy, S.K. On invex sets and preinvex functions. J. Math. Anal. Appl. 1995, 189, 901-908. [CrossRef]

45. Khan, M.B.; Noor, M.A.; Abdeljawad, T.; Abdalla, B.; Althobaiti, A. Some fuzzy-interval integral inequalities for harmonically convex fuzzy-interval-valued functions. AIMS Math 2020, 7, 349-370. [CrossRef]

46. Khan, M.B.; Noor, M.A.; Noor, K.I. On Fuzzy Quasi-Invex Sets. Int. J. Alg. Stat. 2020, 9, 11-26.

47. Khan, M.B.; Noor, M.A.; Noor, K.I. On Some Characterization of Preinvex Fuzzy Mappings. Earth. J. Math. Sci. 2021, 5, 17-42. [CrossRef]

48. Khan, M.B.; Noor, M.A.; Al-Shomrani, M.M.; Abdullah, L. Some Novel Inequalities for LR-h-Convex Interval-Valued Functions by Means of Pseudo Order Relation. Math. Meth. Appl. Sci. 2021. [CrossRef]

49. Khan, M.B.; Noor, M.A.; Noor, K.I.; Almusawa, H.; Nisar, K.S. Exponentially Preinvex Fuzzy Mappings and Fuzzy Exponentially Mixed Variational-Like Inequalities. Int. J. Anal. Appl. 2021, 19, 518-541.

50. Khan, M.B.; Noor, M.A.; Al-Bayatti, H.M.; Noor, K.I. Some New Inequalities for LR-Log-h-Convex Interval-Valued Functions by Means of Pseudo Order Relation. Appl. Math. 2021, 15, 459-470.

51. Khan, M.B.; Noor, M.A.; Noor, K.I.; Chu, Y.M. Higher-Order Strongly Preinvex Fuzzy Mappings and Fuzzy Mixed Variational-Like Inequalities. Int. J. Comput. Intell. Syst. 2021, 14, 1856-1870. [CrossRef]

52. Liu, P.; Khan, M.B.; Noor, M.A.; Noor, K.I. On Strongly Generalized Preinvex Fuzzy Mappings. J. Math. 2021, 2021. [CrossRef]

53. Khan, M.B.; Noor, M.A.; Noor, K.I.; Ab Ghani, A.T.; Abdullah, L. Extended perturbed mixed variational-like inequalities for fuzzy mappings. J. Math. 2021, 2021. [CrossRef]

54. Khan, M.B.; Noor, M.A.; Abdullah, L.; Noor, K.I. New Hermite-Hadamard and Jensen Inequalities for Log-h-Convex FuzzyInterval-Valued Functions. Int. J. Comput. Intell. Syst. 2021, 14, 1-16. [CrossRef] 